\title{
Extrasynaptic Muscarinic Acetylcholine Receptors on Neuronal Cell Bodies Regulate Presynaptic Function in Caenorhabditis elegans
}

\author{
Jason P. Chan, ${ }^{1,3,4}$ Trisha A. Staab, ${ }^{1,3}$ Han Wang, ${ }^{1,3}$ Chiara Mazzasette, ${ }^{1,3}$ Zara Butte, ${ }^{1,3}$ and Derek Sieburth ${ }^{1,2,3}$ \\ ${ }^{1}$ Zilkha Neurogenetic Institute, ${ }^{2}$ Department of Cell and Neurobiology, ${ }^{3}$ Keck School of Medicine, University of Southern California, Los Angeles, California \\ 90033, and ${ }^{4}$ Department of Biology, Juniata College, Huntingdon, Pennsylvania 16652
}

Acetylcholine $(\mathrm{ACh})$ is a potent neuromodulator in the brain, and its effects on cognition and memory formation are largely performed through muscarinic acetylcholine receptors ( $\mathrm{mAChRs).} \mathrm{mAChRs}$ are often preferentially distributed on specialized membrane regions in neurons, but the significance of $\mathrm{mAChR}$ localization in modulating neuronal function is not known. Here we show that the Caenorhabditis elegans homolog of the M1/M3/M5 family of mAChRs, gar-3, is expressed in cholinergic motor neurons, and GAR-3-GFP fusion proteins localize to cell bodies where they are enriched at extrasynaptic regions that are in contact with the basal lamina. The GAR-3 N-terminal extracellular domain is necessary and sufficient for this asymmetric distribution, and mutation of a predicted $N$-linked glycosylation site within the N-terminus disrupts GAR-3-GFP localization. In transgenic animals expressing GAR-3 variants that are no longer asymmetrically localized, synaptic transmission at neuromuscular junctions is impaired and there is a reduction in the abundance of the presynaptic protein sphingosine kinase at release sites. Finally, GAR-3 can be activated by endogenously produced ACh released from neurons that do not directly contact cholinergic motor neurons. Together, our results suggest that humoral activation of asymmetrically localized $\mathrm{mAChRs}$ by $\mathrm{ACh}$ is an evolutionarily conserved mechanism by which ACh modulates neuronal function.

\section{Introduction}

Muscarinic acetylcholine (ACh) receptors are G-protein coupled receptors (GPCRs) that mediate many of the modulatory functions of ACh in the CNS. Muscarinic signaling affects multiple behaviors-including alertness, cognition, memory, and motor control-by altering neuronal excitability, synaptic protein composition, and the strength of communication between neurons and other tissues (Picciotto et al., 2012). The five subtypes of mammalian muscarinic ACh receptors (mAChRs), M1-M5, differ in ligand affinity, subcellular localization, and signaling capacity. Generally, M1, M3, and M5 couple to the G $\alpha \mathrm{q} / 11$ heterotrimeric G-protein, and their activation leads to an increase in neuronal activity; M2 and M4, on the other hand, couple to $\mathrm{G} \alpha \mathrm{i} / \mathrm{o}$ and negatively regulate neurotransmitter release from presynaptic terminals (Eglen, 2006; Wess et al., 2007).

Muscarinic receptor subtypes are differentially distributed on specialized membrane regions within neurons (Levey et al., 1991; Rouse et al., 1998; Oldenburg and Ding, 2011). Immunohisto-

\footnotetext{
Received March 30, 2013; revised July 23, 2013; accepted July 26, 2013.

Author contributions: J.P.C. and D.S. designed research; J.P.C., T.A.S., H.W., C.M., Z.B., and D.S. performed research; J.P.C., T.A.S., and D.S. analyzed data; J.P.C. and D.S. wrote the paper.

This work was supported by grants from the American Heart Association and the National Institutes of Health to D.S. (NS071085). We thank the Caenorhabditis Genetic Center and the National BioResource Project for strains used in this study. We thank B. Ramos, R. Lakshminarasimhan, C. Gillian, and J. Chen for technical assistance. We thank Joshua Kaplan for nuls321.

The authors declare no competing financial interests.

Correspondence should be addressed to Dr. Derek Sieburth, University of Southern California, 1501 San Pablo Street, ZNI321, Los Angeles, CA 90089. E-mail: sieburth@usc.edu.

DOI:10.1523/JNEUROSCI.1359-13.2013

Copyright $\odot 2013$ the authors $\quad 0270-6474 / 13 / 3314146-14 \$ 15.00 / 0$
}

chemical studies of $\mathrm{M} 1$ receptors, for example, show preferential localization on cell bodies and dendrites (Yamasaki et al., 2010), whereas $\mathrm{M} 2$ receptor abundance is generally greater on axon terminals (Rouse et al., 1997). At these specialized membrane regions, mAChRs can be found at either synaptic or extrasynaptic sites. For example, ultrastructural studies show that M2/M4 receptors can localize to extrasynaptic sites of presynaptic terminals, where they can function as autoreceptors or heteroreceptors (Rouse et al., 2000; Picciotto et al., 2012). On the other hand, M1 receptors can localize to extrasynaptic regions on dendritic spines and shafts (Mrzljak et al., 1993; Yamasaki et al., 2010), where they primarily function as heteroreceptors. The highly specialized $\mathrm{mAChR}$ receptor distribution in the brain suggests that receptor localization may be a critical component underlying cholinergic modulation of neuronal physiology.

Extrasynaptic receptors can respond to ACh released from distant sources, such as neurotransmitter that escapes the synapse (spillover) or diffuses through the extracellular space from distant sites (Agnati et al., 2010; Vizi et al., 2010). This signaling, termed "volume transmission," is supported by observations that central cholinergic nerve endings are distributed at lower densities than AChR binding sites, and cholinergic terminals are often not associated with postsynaptic membrane specializations ( $\mathrm{Mr}$ zljak et al., 1993; Descarries and Mechawar, 2000). In volume transmission, one neuron secreting ACh can act on extrasynaptic $\mathrm{mAChRs}$ of many targets to modulate aspects of cholinergic signaling, such as those associated with arousal and attention (Sarter et al., 2009; Vizi et al., 2010; Fuxe et al., 2012). However, the molecular mechanisms underlying mAChR localization and the 
functional importance of $\mathrm{mAChR}$ localization in neurons are still unclear.

Here, we show that a Caenorhabditis elegans mAChR M1/ M3/M5 family member, GAR-3, is asymmetrically enriched on extrasynaptic membrane surfaces of motor neuron cell bodies, and this asymmetric localization is necessary for its effects on neuromuscular function. We find that GAR-3 is in contact with the pseudocoelom, a cavity analogous to the circulatory system, and endogenous $\mathrm{ACh}$ released from a distance can activate GAR-3 on motor neurons. We propose a model whereby ACh released into the pseudocoelom activates GAR-3 to modulate cholinergic neurotransmission.

\section{Materials and Methods}

C. elegans strains. All behavioral experiments were performed on young adult hermaphrodites, unless otherwise noted. Some strains were provided by the Caenorhabditis Genetic Center, which is funded by NIH Office of Research Infrastructure Programs (P40 OD010440). The wild-type reference strain was N2 Bristol. The following strains were used: gar-3(gk305), nre-1(hd20) lin15b(hd126), unc-2(li1), unc-13(s69), unc-17(e245), unc-80(e1272), unc-104(e1265), nuIs197[Pmyo-2::GFP, Punc-129::sphk-1-GFP], nuIs321[Punc-17::mCherry], vjIs50[Pttx-3:mRFP, Punc-129::GAR-3-GFP], vjIs14[Punc-129::mCherry], vjSi06[Punc-129::gar-3-gfp, cb-unc-119(+)]. All strains used were outcrossed $>4$ times and integrated transgenes were outcrossed 10 times.

Molecular biology. The gar-3 promoter fragment was amplified by PCR from mixed stage genomic DNA, and GAR-3 chimeras were made using gar-3b and gar- 1 cDNA. All primer sequences are available upon request. The following plasmids were made by standard molecular biology techniques (plasmid name[promoter-gene-marker]): pDS221[Punc129::sphk-1-gfp], pDS322[Punc-129::gar-3b-gfp], pDS333[Punc-17:: unc-17], pDS324[Punc-17::gar-3b], pDS336[Punc-129::gar-1-gfp], pDS339[Punc-129::gar-3(NT3)-gfp], pDS340[Punc-129::gar-3(2NQ)-gfp], pDS341[Punc-129::gar-1-3-gfp], pDS342[Punc-129::gar-3-1-gfp], pDS343 [Punc-129::gar-3(1i3)-gfp], pDS345[Punc-129::gar-3(N33Q)-gfp], pDS346[Punc-129::gar-3(N28Q)-gfp], pDS347[Punc-17::gar-3b-gfp], pDS348[Punc-129::gar-3(NT1)-gfp], pDS349[Punc-129::gar-3(NT2)$g f p]$, pDS354[Psra-6::mCherry], pDS364[Pgar-3(8.5kB)::gfp], pDS368 [Pgar-3(8.5kB)::gar-3b-gfp], pDS369[Pdfk-2b::mCherry], pDS371[Punc-17:: gar-1], pDS372[Punc-17::gar-1-3b], pDS373[Pmyo-3::gar-3-gfp], pDS374 [Pmyo-3::gar-1-3-gfp], pDS399[Punc-47::mCherry], pDS400[Pglr-1:: $m$ Cherry], pDS401[Punc-17( $\Delta):: m$ Cherry]. pDS402[Punc-17(A)::unc-17]. To make a MosSCI plasmid (pDS404), Punc-129::gar-3b-gfp was cloned into pCFJ151.

Transgenic lines. Transgenic strains were generated by injecting either N2, gar-3( gk305), or nuIs197 animals with expression constructs ( $10-25 \mathrm{ng} / \mu \mathrm{l})$ and a coinjection marker [KP\#708 (Pttx-3::rfp, $40 \mathrm{ng} / \mu \mathrm{l}$ ) or KP\#1106 (Pmyo-2::nls-gfp, $10 \mathrm{ng} / \mu \mathrm{l})$ ]. Microinjection was performed using standard techniques as previously described (Mello et al., 1991). At least three lines for each transgene were examined and a single, representative transgene was used for the experiments. The following transgenic lines were made (name[plasmid]): vjEx579[PDS333], vjEx601[pDS364], vjEx602[pDS368], vjEx603[pDS369], vjEx635[pDS324], vjEx732[pDS322], vjEx733[pDS340], vjEx734[pDS341], vjEx735[pDS342], vjEx736[pDS343], vjEx737[pDS345], $v j E x 738[p D S 346], v j E x 741$ [pDS354], vjEx742[pDS374], vjEx743[pDS371], vjEx744[pDS372], vjEx745[pDS347], vjEx746[pDS399], vjEx747[pDS400], vjEx748[pDS373], vjEx749[pDS348], vjEx750[pDS349], vjEx751[pDS339], vjEx753[pDS336], vjEx754[pDS401], vjEx755[ [pDS402].

Behavioral analysis. For analysis of neurotransmission, animals were tested for their sensitivity to aldicarb (Bayer CropScience), an inhibitor of acetylcholinesterase. Animals paralyze after acute exposure to $1.0 \mathrm{~mm}$ aldicarb, and paralysis of adult worms was scored every $10 \mathrm{~min}$, starting at $40 \mathrm{~min}$. For each experiment, animals were exposed to NGM plates supplemented with aldicarb, using $\sim 20$ worms per plate in triplicate. The number of worms paralyzed on each plate was counted to extract the percentage paralyzed at each time-point per genotype. The percentages were averaged at each time point per genotype and plotted graphically. For each experiment, the genotype was blind to the scorer, and the anal- ysis was repeated at least two times. For experiments using arecoline, worms were pretreated with plates containing either control M9 or arecoline (15 mM in M9) for $2 \mathrm{~h}$. This pretreatment duration was determined to be the minimum concentration that produced maximal aldicarb hypersensitivity, to minimize nonspecific effects of the drug.

RNAi. RNAi feeding was performed as previously described (Kamath et al., 2001). Briefly, RNAi feeding clones were grown for $12 \mathrm{~h}$ at $37^{\circ} \mathrm{C}$ in LB culture supplemented with $50 \mu \mathrm{g} / \mathrm{ml}$ carbenicillin. RNAi clones were then seeded onto agar plates containing IPTG ( $1 \mathrm{mM})$ and carbenicillin $(50 \mu \mathrm{g} / \mathrm{ml})$. Eggs were isolated from adult state worms [vjIs50[Punc-129::gar-3b-gfp];nre-1(hd20) lin-15b(hd126)] using a sodium hypochlorite solution. Eggs were washed with M9, and $\sim 100$ eggs were added to each seeded RNAi plate. After 3-4 d, animals were removed from bacteria and assessed for GAR-3-GFP localization. To ensure effective RNAi, we screened animals fed the gar-3 RNAi clone, as a positive control, which resulted in a $64 \%$ reduction in fluorescence compared with feeding with an empty RNAi vector (L4440). For each RNAi treatment, we determined the number of motor neurons in which GAR3-GFP adopted a uniform fluorescence distribution. Approximately $11.3 \%(6 / 53)$ of cells examined in animals not treated with RNAi expressed nonlocalized GAR-3-GFP. Statistical differences were determined by $\chi^{2}\left(\psi^{2}\right)$ test, and significance was set at $p<0.05$. Results are reported in Table 1.

Microscopy and analysis. Fluorescence microscopy experiments were performed as previously described (Ch'ng et al., 2008). Briefly, adult worms (unless otherwise stated) were paralyzed using 2,3-Butanedione monoxime ( $30 \mu \mathrm{g} / \mu \mathrm{l}$, Sigma-Aldrich) and mounted on $2 \%$ agarose pads for imaging. For all fluorescence microscopy experiments, images were captured with a Nikon eclipse $90 i$ microscope equipped with a Nikon PlanApo $100 \times$ objective $(\mathrm{NA}=1.4)$ and a Photometrics Coolsnap ES ${ }^{2}$ camera. MetaMorph 7.0 software (Universal Imaging; Molecular Devices) was used to analyze images.

For analyses of GAR-3-GFP on cell bodies, images were captured from the ventral motor neurons throughout the anterior-posterior axis of the animals. For quantification of asymmetry, pixel intensities were recorded manually (using MetaMorph), and the background fluorescence was subtracted. For quantification of total GAR-3-GFP membrane fluorescence, pixel values were recorded along the surface of the neuronal cell body manually (using MetaMorph), averaged, and the background fluorescence was subtracted for the final fluorescence value. For treatment of animals with drugs, animals were dissected as described previously (Richmond, 2006) to expose the extracellular matrix (ECM). Either control solution (M9), collagenase type IV (Sigma-Aldrich), or trypsin (Cellgro) were added to the worm before analysis of GAR-3-GFP. For colocalizing GAR-3-GFP with wheat germ agglutinin (WGA; Alexa Fluor 594; Invitrogen), the WGA conjugate was injected into the pseudocoelomic region, allowed $15 \mathrm{~min}$ for recovery, and analyzed. For analysis following RNAi treatment, DA/DB cells throughout the $\mathrm{A} / \mathrm{P}$ axis of the animal were scored as either asymmetrical or uniform.

For analysis of SPHK-1-GFP at synapses, serial image stacks were captured from dorsal axons of DA/DB neurons near the posterior gonadal bend of adult worms, and the maximum intensity projection was used for analysis of the dorsal cords. Line scans of the maximum intensity projection image were also recorded using MetaMorph. The fluorescence intensity values were then quantified using Puncta 6.0 software written with Igor Pro (Wavemetrics), as previously described (Dittman and Kaplan, 2006; Ch'ng et al., 2008). Quantification spanned $\sim 500$ synapses for all experiments, and specific $n$ values are labels in graphs. For all experiments, fluorescence values were normalized to the values of 0.5 $\mu \mathrm{m}$ FluoSphere beads (Invitrogen) captured during each imaging session. This was performed to provide a standard for comparing absolute fluorescence levels between animals from different sessions. We found that the conditions used for imaging showed that SPHK-1-GFP were predominantly localized to synaptic puncta, as determined previously (Chan et al., 2012). There was little or no difference between their interpunctal fluorescence (in axons) and the auto-fluorescence observed at $C$. elegans axons. Therefore, we excluded the interpunctal fluorescence in our analyses of SPHK-1-GFP. 
Table 1. RNAi analysis of GAR-3-GFP asymmetry

\begin{tabular}{|c|c|c|c|c|c|}
\hline C. elegans gene & Cosmid & Protein ortholog & \% Cells uniform & Total cells examined & $\psi^{2}$ \\
\hline \multicolumn{6}{|l|}{ Cell polarity } \\
\hline$|g|-1$ & F56F10.4 & Lethal giant larvae & 27.3 & 22 & 2.944 \\
\hline lin-2 & F17E5.1a & (MAGUK) family & 6.7 & 30 & 0.476 \\
\hline mom-5 & T23D8.1 & Frizzled receptor & 7.1 & 28 & 0.359 \\
\hline$m i g-5$ & T05C12.6 & Disheveled & 8.3 & 24 & 0.158 \\
\hline par-1 & H39E23.1a & Serine-threonine kinase & 10.0 & 50 & 0.047 \\
\hline Unknown & C25A8.5 & FER kinase & 5.6 & 18 & 0.503 \\
\hline vang-1 & B0410.2 & Strabismus & 7.1 & 28 & 0.359 \\
\hline \multicolumn{6}{|l|}{ Glycosylation } \\
\hline aman-3 & $\mathrm{F} 48 \mathrm{C} 1.1$ & Processing glycosydase & 13.8 & 29 & 0.107 \\
\hline dad-1 & F57B10.10 & Oligosaccharyltransferase complex & 11.4 & 35 & 0.000 \\
\hline$g l y-13$ & B0416.6 & $\mathrm{N}$-acetylglycosaminyl-transferase & 23.3 & 30 & 2.093 \\
\hline Unknown & F41D3.6 & Galectin & 22.9 & 48 & 2.419 \\
\hline Unknown & C47E12.3 & $\alpha 1,2$ mannosidase I & 15.0 & 20 & 0.182 \\
\hline Unknown & F13H10.4 & $\alpha 1,2$ glycosidase I & 11.8 & 17 & 0.003 \\
\hline Unknown & $\mathrm{F} 26 \mathrm{H} 9.8$ & Glycoprotein GICT & 17.4 & 23 & 0.517 \\
\hline Unknown & ZC506.1 & Processing glycosydase & 5.4 & 37 & 0.941 \\
\hline Unknown & T03G11.4 & Processing glycosydase & 14.0 & 43 & 0.150 \\
\hline Unknown & ZK686.3 & Oligosaccharyltransferase complex & 6.5 & 31 & 0.538 \\
\hline Unknown & $\mathrm{T} 12 \mathrm{~A} 2.2$ & Oligosaccharyltransferase complex & 12.8 & 39 & 0.048 \\
\hline Unknown & $\mathrm{T} 19 \mathrm{~B} 12.6$ & Heparin sulphate & 18.2 & 33 & 0.797 \\
\hline
\end{tabular}

GAR-3-GFP was analyzed following RNAi knockdown of select genes involved in cell polarity and glycosylation. The effect of the RNAi treatment was calculated by scoring the number of non-asymmetric cells (equal fluorescence throughout the motor neuron cell body) over the total number of cells examined. We analyzed the neurons throughout the A/P axis, as depicted in Figure 2 . A $\chi^{2}$ test was performed based on the number of uniform cells observed in WT animals ( $6 / 53$, $11.3 \%)$.

*Significant genes $(p<0.05)$.

Statistical analysis. A Student's $t$ test was used to determine significance when comparing the fluorescence of SPHK-1-GFP or GAR-3GFP in different conditions (significance was set at $p<0.05$ and determined by Student's $t$ tests). A $\chi^{2}$ test was performed to determine the significance of GAR-3-GFP asymmetry in the RNAi experiments. SEM (SEM) was performed for all comparisons and displayed graphically. Quantification of synaptic marker abundance at puncta (punctal fluorescence) and synaptic marker density in axons (density) were performed as previously described (Ch'ng et al., 2008) and are useful measures of the structural and functional properties of presynaptic terminals.

\section{Results}

\section{gar-3 functions in cholinergic motor neurons to regulate} aldicarb responsiveness

GAR-3 signaling regulates locomotion and the recruitment of SPHK-1/sphingosine kinase to presynaptic terminals of cholinergic neurons at neuromuscular junctions (NMJs; Dittman and Kaplan, 2008; Chan et al., 2012). To determine in which cells GAR-3 acts to regulate NMJ function, we investigated the expression pattern of a transcriptional gar-3 reporter $(P$ gar-3::gfp $)$ in which an $8.5 \mathrm{~kb}$ promoter fragment immediately upstream of the gar-3 start site drives the expression of $g f p$. This promoter fragment is longer than promoters previously used to examine gar-3 localization (Fig. 1A; Steger and Avery, 2004; Liu et al., 2007). In transgenic animals expressing $P$ gar-3::gfp, fluorescence was detected in body wall and pharynx muscles, as well as in motor neurons of the ventral nerve cord (Fig. $1 A, B$ and data not shown). GFP fluorescence was detected in all cholinergic motor neurons of the ventral nerve cord (labeled with mCherry) but not in GABAergic motor neurons (Fig. 1B).
To determine whether GAR-3 functions in cholinergic motor neurons or in body wall muscles to regulate NMJ function, we examined the responses of gar-3 mutants to drugs that promote neurotransmission at NMJs. Aldicarb is an acetylcholine esterase inhibitor that causes the accumulation of ACh at cholinergic synapses, which leads to muscle hypercontraction and paralysis (Miller et al., 1996; Mahoney et al., 2006). Conditions or mutations that delay aldicarb-induced paralysis (resistance to aldicarb) generally reduce ACh release from motor neurons or reduce the excitability of body wall muscles. Conversely, conditions or mutations that accelerate the rate of aldicarbinduced paralysis (hypersensitivity to aldicarb) generally have increased neurotransmitter release or enhanced muscle excitability (Miller et al., 1996; Mahoney et al., 2006). gar-3(gk305) mutants, which have a 452 nucleotide deletion predicted to remove the C-terminal 153 aa of the receptor, were resistant to aldicarb compared with wild-type controls (Fig. 1C; Chan et al., 2012). Expression of full-length gar-3b cDNA in cholinergic motor neurons (using the $u n c-17$ promoter) caused gar-3 mutants to become hypersensitive to aldicarb, whereas expression of gar- $3 b$ cDNA in muscles (using the myo-3 promoter) did not cause aldicarb hypersensitivity (Fig. 1C,D).

The nicotinic agonist levamisole activates receptors that cause body wall muscle contraction, and levamisole treatment results in paralysis due to muscle hypercontraction (Lewis et al., 1980). The rate of paralysis of gar-3 mutants treated with levamisole was similar to that of wild-type controls, and expression of gar-3 cDNA in muscles did not alter responses of gar-3 mutants to levamisole (Fig. 1E). Together, these data suggest that gar-3 mu- 
A

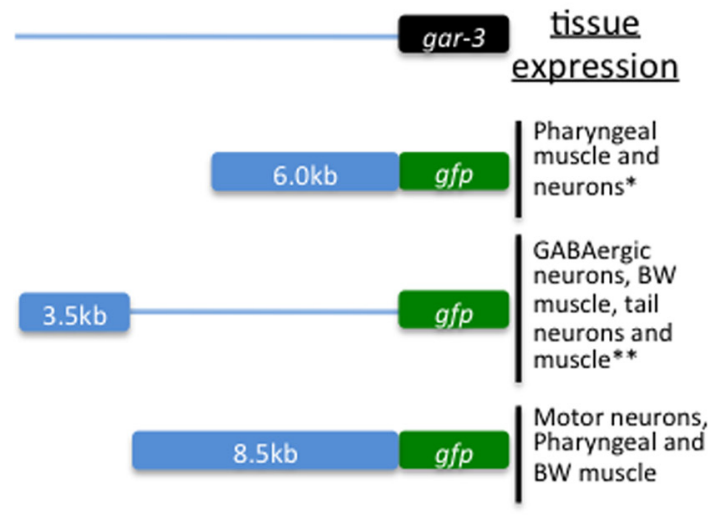

C

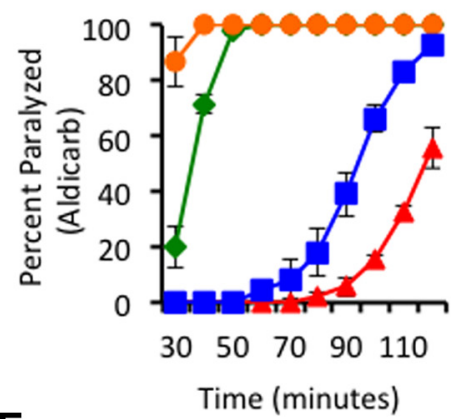

E

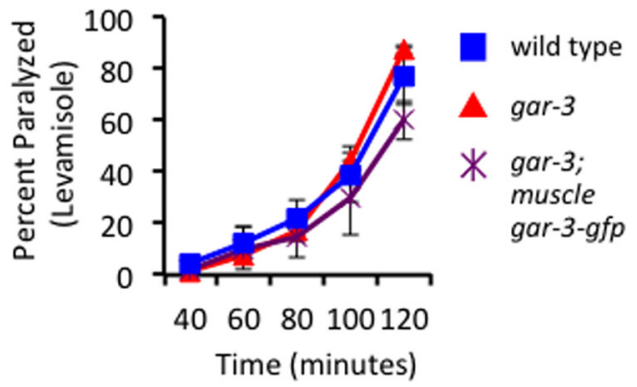

B

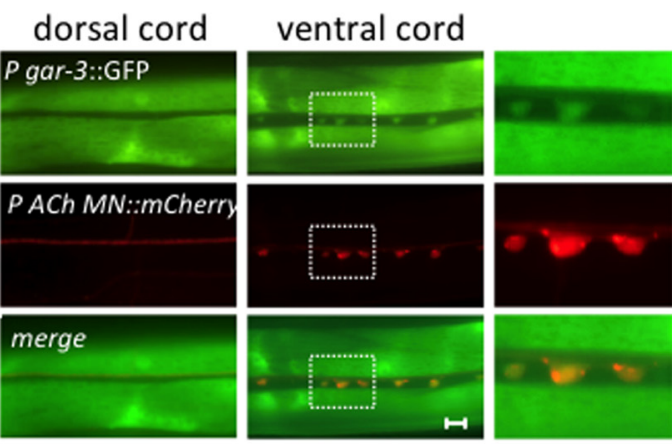

D

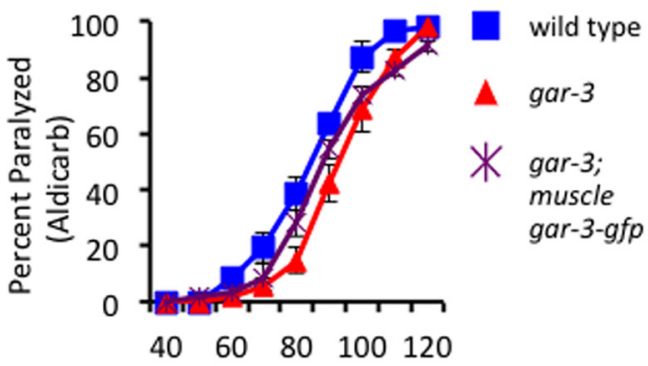

Time (minutes)

A gar-3

gar-3;

gar-3;

cholinergic gar-3-gfp 
A
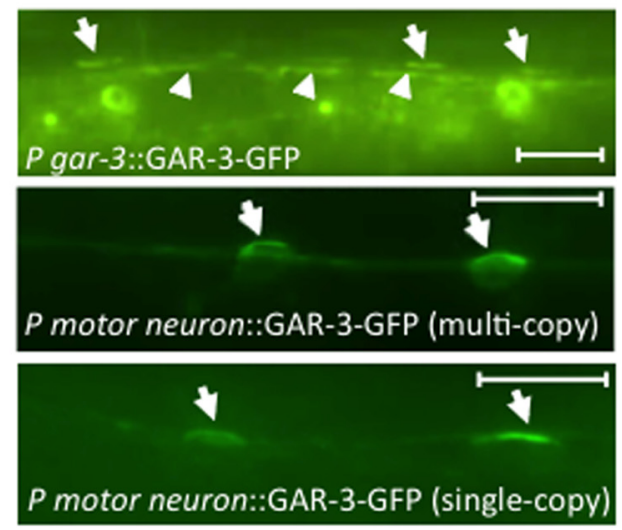

C
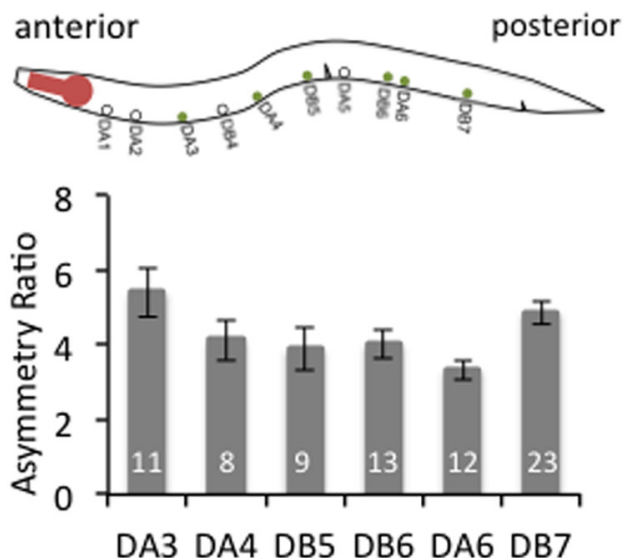

E
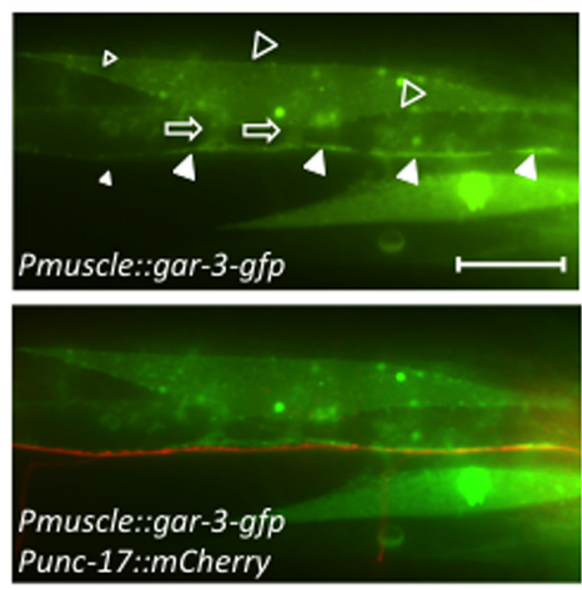

B
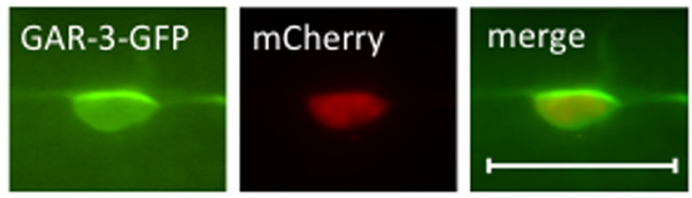

D

GAR-3-GFP in cholinergic motor neurons
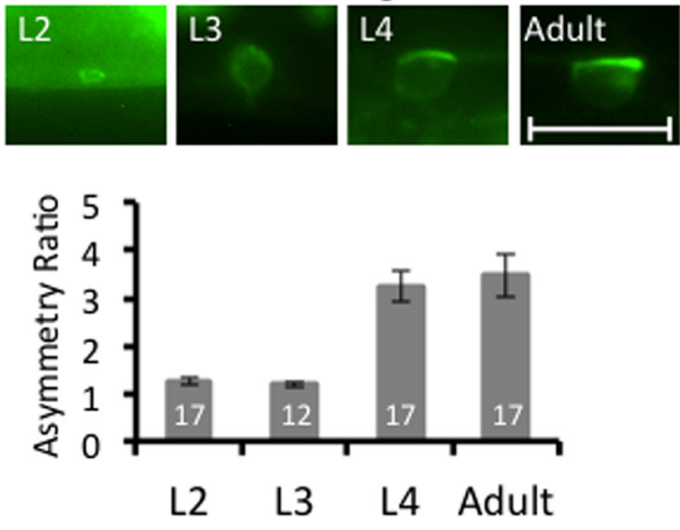

$\mathbf{F}$
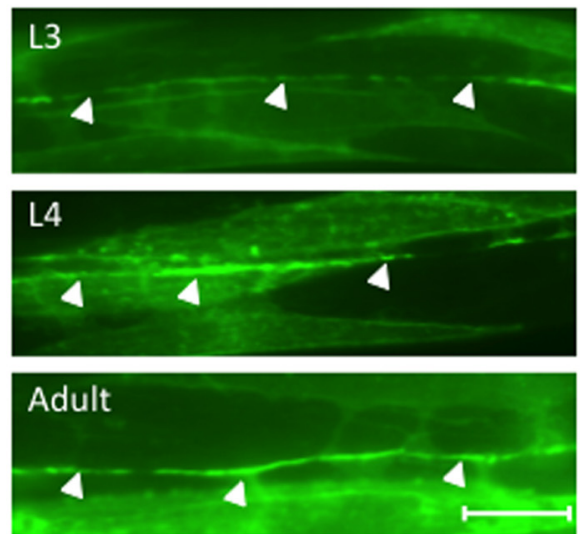

Figure 2. GAR-3/mAChR is asymmetrically localized on membranes of motor neurons and body wall muscles. $A$, Images showing the localization of GAR-3-GFP expressed under its endogenous promoter (vjEx602[P gar-3(8.5)::gar-3b-gfp)], as a multicopy integrant under a motor neuron promoter (vjls50[P unc-129::gar-3b-gfp]), or as a single copy integrant under a motor neuron promoter vjsi06[P unc-129::gar-3b-gfp]). B, Representative images showing the localization of GAR-3-GFP (vjls50) and mCherry (vjls14[P unc-129::mCherry]) in DA/DB cell bodies. C, Top, Diagram and quantification of the DA/DB neurons examined along the A/P axis of the worm. Cells examined in this study are filled-in circles. $D$, Representative images and quantification of GAR-3-GFP localization in motor neurons (vjls50) from L2 to adult stages. E, Representative images showing the colocalization of GAR-3-GFP in body wall muscles (vjEx748[P myo-3::gar-3b-gfp]) with mCherry expressed in cholinergic neurons (nuls321[P unc-17::mCherry]). $\boldsymbol{F}$, Representative images showing GAR-3-GFP in muscles (vjEx748) of L3 to adult stages. $\boldsymbol{A}, \boldsymbol{E}, \boldsymbol{F}$, Filled arrows indicate motor neuron cell bodies, filled arrowheads indicate postsynaptic membranes of in the dorsal nerve cord, open arrowheads indicate muscle cell membranes, and open arrows indicate muscle arms. $\boldsymbol{C}, \boldsymbol{D}$, Numbers in bars represent the number of animals examined and error bars indicate \pm SEM. Scale bars, $10 \mu \mathrm{m}$.

To quantify the asymmetric distribution of GAR-3-GFP in neuron cell bodies, we compared fluorescence on the dorsal and ventral sides of cell bodies to establish an asymmetry ratio $(\mathrm{D} / \mathrm{V})$. The asymmetry ratio in DA/DB class neurons expressing multicopy GAR-3-gfp was $4.9 \pm 0.3$ (expressed as mean \pm SEM hence- forth, $n=53)$. In neurons expressing single-copy GAR-3-gfp in $\mathrm{DA} / \mathrm{DB}$ class, the ratio was $6.2 \pm 0.9(n=17)$. GAR-3-GFP was asymmetrically distributed in each of the DA/DB class neurons examined along the anterior-posterior length of the ventral nerve cord (Fig. 2C). GAR-3-GFP fluorescence was not detected in ax- 
ons in single-copy transgenic animals (0 of 15 axons) and was rarely detected in axons of multicopy animals ( 3 of 23 axons). GAR-3-GFP was also not detected in dendrites of single-copy transgenic animals, and was occasionally detected in proximal dendrites of multicopy animals (data not shown). When axonal or dendritic GAR-3-GFP fluorescence was detected, it adopted a diffuse pattern of localization (Fig. $2 A, B$, and data not shown). Based on these results, we conclude that GAR-3 is highly enriched in cholinergic motor neuron cell bodies, where it asymmetrically distributes to the dorsal surfaces, but is likely to be very low or absent from neuronal processes.

GAR-3-GFP localization was not asymmetric early in development (asymmetry ratio $1.19 \pm 0.6$ in the L3 stage), but it became asymmetric starting at the L4 stage $(3.25 \pm 0.34)$ and maintained asymmetry in adults (Fig. 2D). GAR-3-GFP on muscle membranes was visible in young animals (L3 stage) and appeared enhanced in older animals (Fig. $2 F$ ). These observations suggest that the asymmetric localization of GAR-3 in motor neuron cell bodies and muscles may be under developmental control.

\section{The N-terminal extracellular domain of GAR-3 is necessary and sufficient for asymmetric localization}

To investigate mechanisms underlying GAR-3 asymmetry, we first examined the localization of chimeras in which domains of GAR-3 were replaced with homologous domains from another mAChR family member, GAR-1. Unlike GAR-3, GAR-1-GFP fusion proteins adopted a uniform (symmetric) localization pattern in motor neuron cell bodies (asymmetry ratio of $1.20 \pm 0.04$; Fig. $3 A, B)$. The third intracellular domain of GPCRs, the i3 loop, specifies G-protein coupling and has been shown to be important for GPCR trafficking (Nathanson, 2008). GAR-3 chimeras containing the intracellular loop of GAR-1 [GAR-3(1-i3)] were similarly asymmetric as GAR-3-GFP (4.92 \pm 0.95$)$, suggesting that the i3 loop is not necessary for GAR-3 asymmetry. Chimeras in which the N-terminal domain of GAR-3 was replaced with the GAR-1 N-terminus (GAR-1-3 chimeras) adopted a uniform distribution (asymmetry ratio of $1.11 \pm 0.05$ ), indistinguishable from that of GAR-1 (Fig. $3 A, B$ ). On the other hand, chimeras in which the N-terminal region of GAR-1 was replaced with that of GAR-3 (GAR-3-1 chimeras) adopted a highly asymmetric distribution $(4.49 \pm 0.70)$. Together these results show that the N-terminus of GAR-3 is both necessary and sufficient for the asymmetric localization of GAR-3-GFP in neuronal cell bodies.

The reduced asymmetrical distribution of the GAR-1-3 chimera was not a secondary effect of a reduction in expression or stability of the chimera: First, in three independently generated transgenic lines, total fluorescence on cell-body surfaces was similar in GAR-3-GFP and GAR-1-3-GFP-expressing animals (Fig. $3 C)$. Second, GAR-1-3-GFP fluorescence appeared to be higher in axons (16 of 21 axons examined) compared with GAR-3-GFP. Thus, the uniform distribution of the GAR-1-3-GFP chimera in cell bodies cannot be explained by a reduction in its expression. The GAR-1-3 chimera also adopted a uniform distribution in body wall muscles, with no enrichment in muscle arms or in postsynaptic membranes of the ventral nerve cord, suggesting that the N-terminus of GAR-3 is required for asymmetric localization in both neurons and muscles (Fig. 3D).

To further map the sequence determinants for GAR-3 localization, we analyzed the cell body distribution of GAR-3-GFP variants with different $\mathrm{N}$-terminal deletions. GAR-3-GFP variants lacking amino acids $13-23$ [GAR-3 $(\Delta \mathrm{NT} 1)]$ or amino acids 24-39 [GAR-3( $\Delta \mathrm{NT} 2)$ ] adopted a uniform pattern of localiza- tion (asymmetry ratios $1.10 \pm 0.03$ and $1.13 \pm 0.04$, respectively), whereas deletion of amino acids 54-66 [GAR-3( $\Delta$ NT3)] remained asymmetrically distributed $(2.94 \pm 0.39$; Fig. $3 A, B)$. Thus, residues between positions 13 and 39 are necessary for the asymmetrical distribution of GAR-3 in neuron cell bodies.

\section{Glycosylation contributes to asymmetric GAR-3 localization}

Two predicted $N$-glycosylation sites, N28 and N33, are present within the N-terminal extracellular region required for GAR-3 asymmetry (consensus: N,X,S/T). Because glycosylation regulates the post-translational processing of mammalian muscarinic receptors and influences receptor trafficking (Liles and $\mathrm{Na}$ thanson, 1986), we examined the effects of altering these sites on GAR-3 localization. GAR-3-GFP variants carrying the N33Q substitution were significantly less asymmetric than wild-type GAR-3-GFP (2.59 \pm 0.26$)$. Variants containing the N28Q substitution, however, remained asymmetric (3.41 \pm 0.38 ; Fig. $3 A, B)$. GAR-3-GFP variants with mutations in both asparagines (N28Q and N33Q) adopted similar reductions in asymmetry as GAR-3-GFP(N33Q; $2.57 \pm 0.31)$. Thus, glycosylation at N33 contributes to GAR-3 asymmetry, but additional N-terminal determinants must also specify GAR-3 localization.

To further explore the contribution of glycosylation on GAR-3 localization, we knocked down enzymes involved in protein glycosylation by RNA interference (RNAi; Table 1). We examined 16 genes predicted to be involved in various aspects of biosynthesis, transfer, and modification of glycosyl groups in the ER. We found that knock down of one gene, ostd-1, the $\delta$ subunit of an oligosaccharyltransferase (OST) complex protein, decreased GAR-3-GFP asymmetry (Table 1). OST proteins participate in protein $\mathrm{N}$-glycosylation by catalyzing the transfer of branched oligosaccharides to Asn residues on nascent polypeptides in the ER (Chavan and Lennarz, 2006).

We next examined other cellular pathways that might regulate GAR-3 asymmetric localization. GPCR receptor activation often leads to a reduction in their cell-surface expression through receptor internalization or redistribution (Ritter and Hall, 2009; Reiner and Nathanson, 2012). Acutely increasing ACh levels with aldicarb, which causes the accumulation of ACh at cholinergic synapses (Mahoney et al., 2006), did not significantly change the GAR-3-GFP asymmetry ratio compared with nontreated controls (Fig. 4A). Similarly, neither genetically decreasing ACh biosynthesis (either acutely in temperature-sensitive mutants for cha-1/choline acetyltransferase or chronically in unc-17/ VAChT mutants) nor ACh release (in $u n c$-2/VGCC) significantly altered the asymmetry ratios (Fig. $4 A, B$ ). Given these results, it seems likely that GAR-3 asymmetry is not regulated by activitydependent mechanisms.

The planar cell polarity (PCP) and Wnt signaling pathways regulate cell polarity by establishing asymmetry on specific regions of cell membranes (Hardin and King, 2008; Nance and Zallen, 2011). To explore whether these pathways regulate GAR-3 distribution, we examined the effects of RNAi-mediated knockdown of PCP or Wnt pathway genes on GAR-3-GFP asymmetry (Table 1). Although, RNAi of most PCP and Wnt pathway genes tested did not generate a significant change in GAR-3-GFP distribution (Table 1), we observed that RNAi of mom-2, one of five secreted Wnt ligands (Green et al., 2008), resulted in a significant reduction of GAR-3-GFP asymmetry (Table 1). Together these results suggest that extracellular determinants, including glycosylation, and possibly specific Wnt signaling pathways, appear to play a role in the establishment or maintenance of asymmetric GAR-3 in neuronal cell bodies. 
A
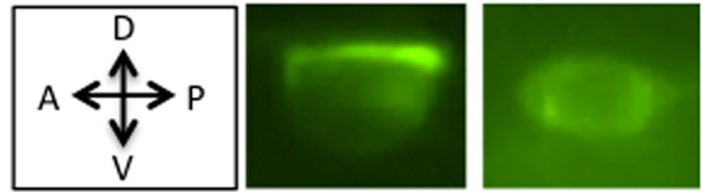

GAR-3
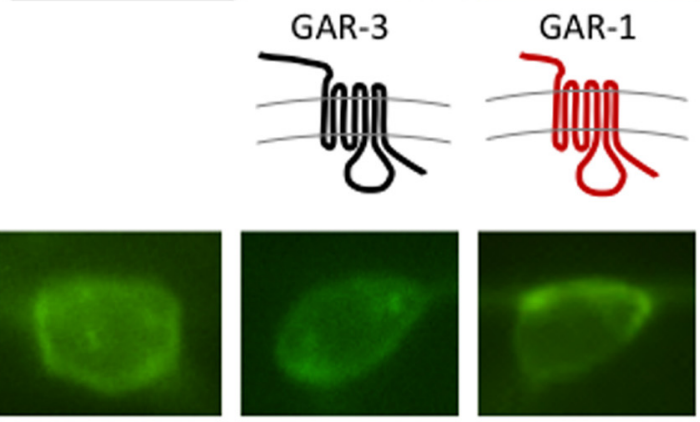

GAR-3( $\Delta N T 1)$

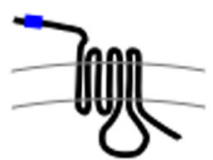

B
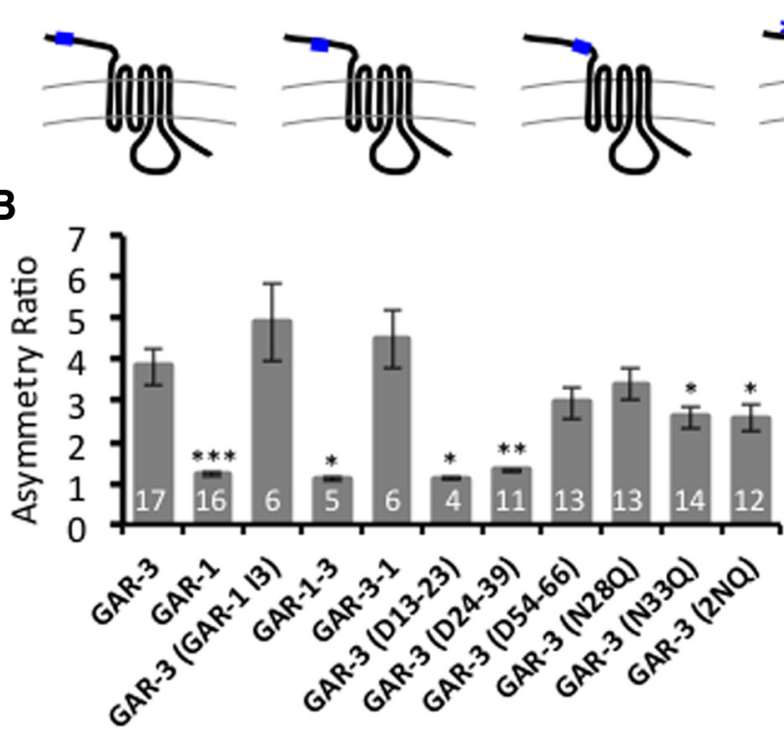

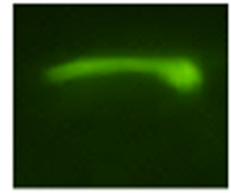

GAR-3(1-i3)
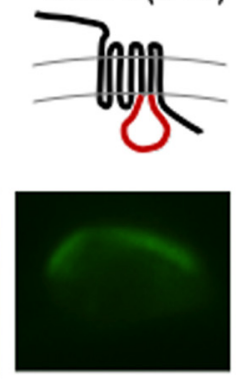

GAR-3(N28Q)
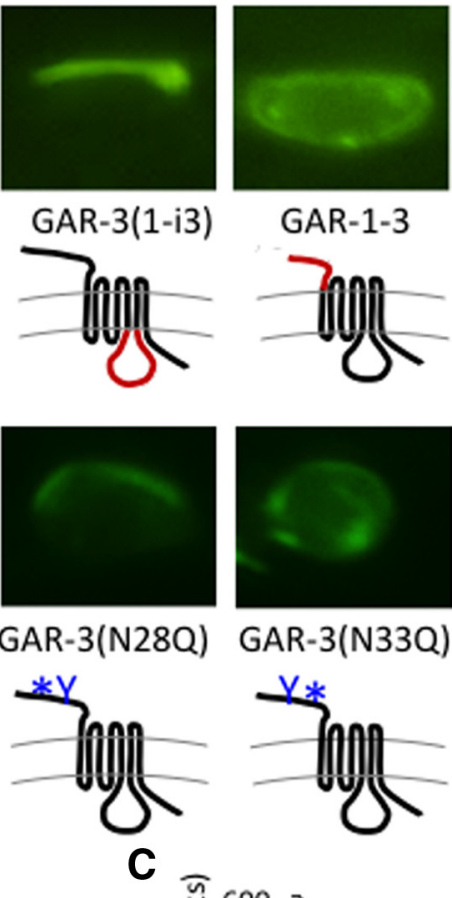

GAR-1-3
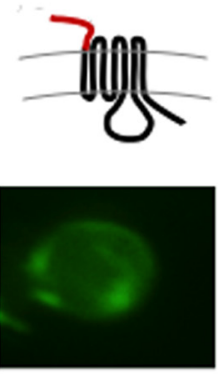

GAR-3(N33Q)
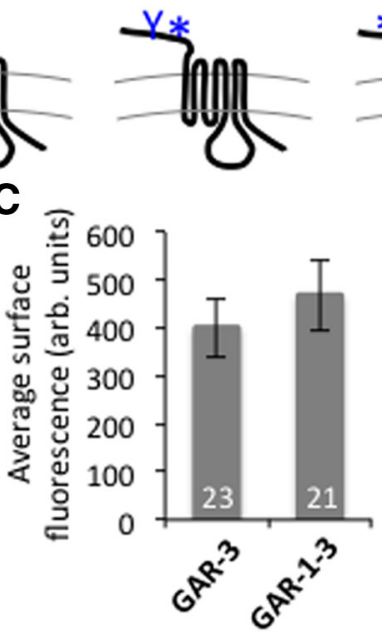

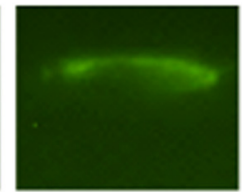

GAR-3-1
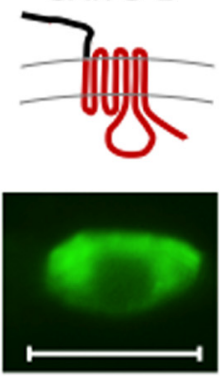

GAR-3(2NQ)

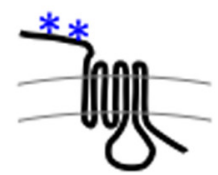

D

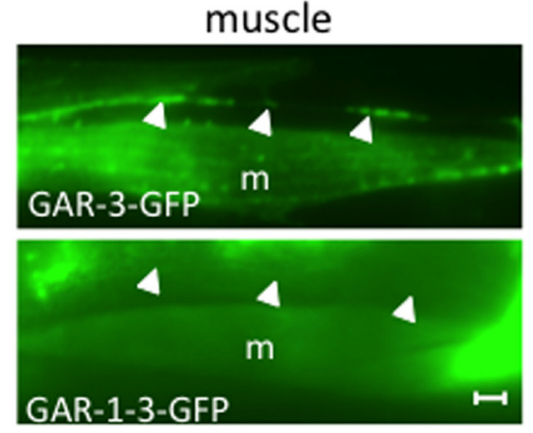

Figure 3. Structural determinants that localize GAR-3/mAChR to restricted membrane regions of neuronal cell bodies. $A, B$, Representative images and quantification of the asymmetry ratio of GAR-3-GFP and GAR-3-GFP variants in DA/DB motor neuron cell bodies. C, Average fluorescence on outer edge of DA/DB cell bodies of GAR-3-GFP and GAR-1-3 chimeras (three independent lines each). D, Representative images of GAR-3-GFP (viEx748) and GAR-1-3-GFP (vjEx742) in body wall muscles. " $\mathrm{m}$ " denotes muscle and arrowhead denotes the ventral nerve cord. $\boldsymbol{A}$, Asterisks denote mutated glycosylation sites. $\boldsymbol{B}, \boldsymbol{C}$, Numbers in bars represent the number of animals examined and error bars are \pm SEM. $\boldsymbol{B},{ }^{*} p<0.05,{ }^{* *} p<0.005,{ }^{* * *} p<0.0005$; Student's t tests. Scale bar, $5 \mu \mathrm{m}$.

Localization of GAR-3 to the basal lamina of the pseudocoelom

We reasoned that interactions between the N-terminus of GAR-3 and adjacent tissues might be involved in GAR-3 localization. 3 -D reconstructions from serial electron microscopy show that the cell bodies of all ventral cord motor neurons are in direct contact with skin (ventral hypodermal ridge), neuronal processes, and the basal lamina of the pseudocoelom (David Hall,
Albert Einstein College of Medicine, New York, personal communication; White et al., 1976). Reconstructions of the DA3 and DA7 motor neurons shows that the skin contacts a large area of the cell bodies along their ventral and medial surfaces (Fig. 5A; White et al., 1976). Several neuronal processes running longitudinally along the ventral nerve cord, including those of DVB, AVF, and PVQ, contact the DA7 cell body along its lateral surface. Finally, the basal lamina is a small region of the ECM that sepa- 
A
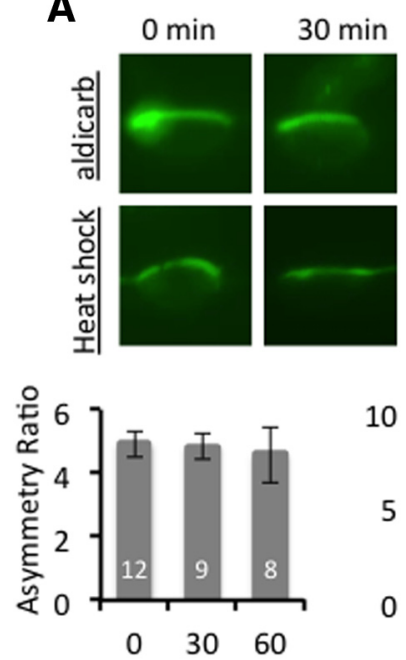

Duration in $1.0 \mathrm{mM}$ aldicarb (minutes)

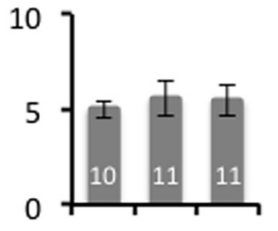

$030 \quad 60$

Duration of heatshock (minutes)
B
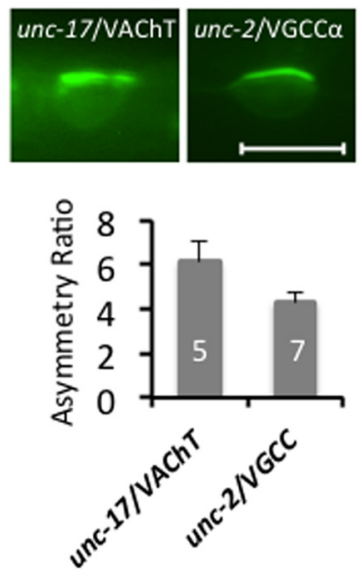

Figure 4. GAR-3 asymmetry is not affected by changes in synaptic ACh levels. $A$, Representative images (top) and quantification (bottom) showing the localization of GAR-3-GFP in DA/DB motor neurons (vj/s50) following 0, 30, and 60 min of aldicarb treatment or heat shock. $\boldsymbol{B}$, Representative images (top) and quantification (bottom) showing the localization of GAR-3-GFP in DA/DB motor neurons (vjls50) in the indicated mutants. Scale bars, $5 \mu \mathrm{m}$. Numbers in bars denote the number of animals examined, and error bars are \pm SEM.

rates the pseudocoelomic cavity from the dorsal surface of motor neuron cell bodies.

Because GAR-3-GFP localized to narrow, dorsal regions of DA/DB cell bodies, it seemed most likely that GAR-3 contacts either one of the dorsally positioned neuronal processes or the basal lamina of the pseudocoelom. We found that GAR-3-GFP fluorescence did not colocalize with the processes of mCherrylabeled DVB, AVF, AVB, or PVQ neurons (Fig. 5A). However, GAR-3-GFP consistently colocalized with rhodamine-conjugated wheat germ agglutinin (WGA) that had been injected into the pseudocoelom. WGA binds to sugar moieties in the ECM, and WGA-labeled membranes appeared as a fluorescent band that lined the pseudocoelom (Fig. 5A). Together, these results suggest that GAR-3 is associated with the basal lamina. Consistent with this, when animals were dissected to expose the DA/DB cell bodies, acute (1 min) treatment with trypsin or collagenase caused GAR-3-GFP to become more uniformly distributed on the surface of the cell body (Fig. $5 B$ ). In the case of trypsin, the asymmetry ratio was significantly reduced from $6.48 \pm 1.05$ in controls to $2.05 \pm 0.44$ in trypsin treated animals $(p<0.05)$. Together these results suggest that GAR-3 is not in contact with neuronal processes in the ventral cord, but rather contacts the basal lamina separating the pseudocoelomic cavity and motor neuron cell body.

\section{Asymmetric localization of GAR-3 is necessary for neuromuscular function}

To address whether the asymmetric localization of GAR-3 in motor neuron cell bodies is important for synaptic function, we examined the effects of mislocalized GAR-3 variants on aldicarb responses and SPHK-1/sphingosine kinase recruitment. The GAR-1-3 chimera is composed of the N-terminal extracellular domain of GAR-1 fused to GAR-3 at the first TM domain (Fig. $3 A$ ). Structure-function studies of mammalian mAChRs have found that the $\mathrm{N}$-terminal extracellular domain does not impact agonist binding, receptor dimerization, or G-protein coupling

(Romero-Fernandez et al., 2011; Leach et al., 2012). Expression of the GAR-1-3 chimera in motor neurons failed to rescue the aldicarb resistance of gar-3 mutants. In contrast, GAR-3 expression in gar-3 mutants caused aldicarb hypersensitivity (Fig. 6A).

Arecoline is a muscarinic agonist that causes gar-3-dependent aldicarb hypersensitivity (Fig. 6A; Lackner et al., 1999; Chan et al., 2012). Arecoline sensitivity was restored to gar-3 mutants by cholinergic expression of wild-type GAR-3 (Fig. $6 A)$. We found that animals expressing GAR-1-3 were as responsive to arecoline treatment as GAR-3-expressing animals since the shifts in aldicarb hypersensitivity following arecoline treatment were similar (Fig. 6A). We conclude that the GAR1-3 chimeras retain normal agonist responses but they fail to restore aldicarb responsiveness to gar-3 mutants because they are not asymmetrically localized.

We next examined the ability of the mislocalized GAR-1-3 chimera to recruit SPHK-1/sphingosine kinase to presynaptic terminals. Previous studies have shown that gar-3 signaling in cholinergic motor neurons increases the synaptic abundance of SPHK-1-GFP fusion proteins. SPHK-1-GFP adopts a punctate pattern of fluorescence in axons, and each puncta represents SPHK-1 at a release site (Chan et al., 2012). Cholinergic GAR-3 expression in gar-3 mutants increased SPHK-1-GFP punctal fluorescence by $50 \%$ compared with controls (Fig. 6B), without altering the number of SPHK-1-GFP puncta $(2.57 \pm 0.08$ puncta per $10 \mu \mathrm{m}$ in control vs $2.46 \pm 0.07$ in gar -3 expressed, $p>0.05$ ). In contrast, cholinergic expression of the mislocalized GAR-1-3 chimera in gar-3 mutants did not significantly increase SPHK-1-GFP abundance (Fig. $6 B)$. Thus, asymmetrically localized GAR -3 is necessary for both the behavioral and synaptic functions mediated by neuronal GAR-3 signaling.

\section{Axonal calcium influx is necessary for GAR-3-mediated SPHK-1 recruitment}

If the activation of GAR-3 on cholinergic neuron cell bodies leads to changes in presynaptic properties, we predict that disrupting signal propagation along axons would block the effects of GAR-3 activation on presynaptic function. In C. elegans, the voltageinsensitive cation leak channel, NALCN (encoded by $n c a-1$ and $n c a-2)$, is required for calcium propagation along axons from cell bodies to nerve terminals (Yeh et al., 2008). unc-80 encodes a conserved NALCN-interacting protein required for the function of the NALCN channel. unc- 80 is expressed in ventral cord motor neurons, and $u n c-80$ mutants have defects in calcium propagation along motor axons (Yeh et al., 2008). In mutants lacking unc-80, steady-state SPHK-1-GFP punctal fluorescence was slightly reduced. Activating GAR-3 by acute arecoline treatment results in gar-3-dependent increases in SPHK-1-GFP punctal fluorescence (Fig. 7A; Chan et al., 2012). The arecoline-mediated increase in SPHK-1-GFP punctal fluorescence was abolished in unc-80 mutants (Fig. 7A). These results show that recruitment of SPHK-1 to synapses by muscarinic signaling requires calcium propagation along the axon. 
A
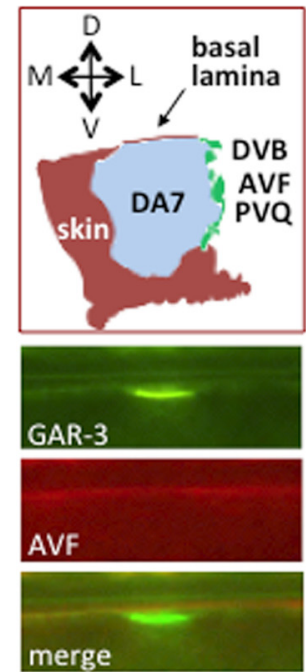
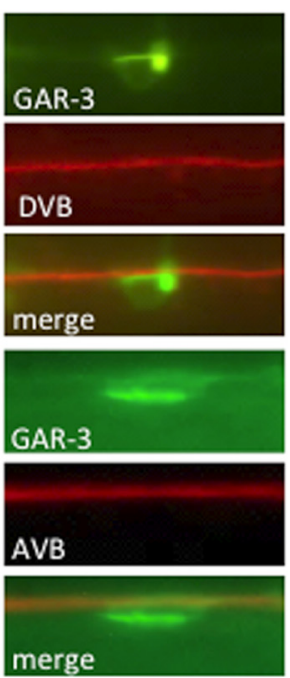

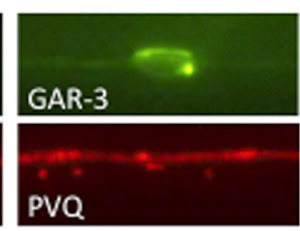

B
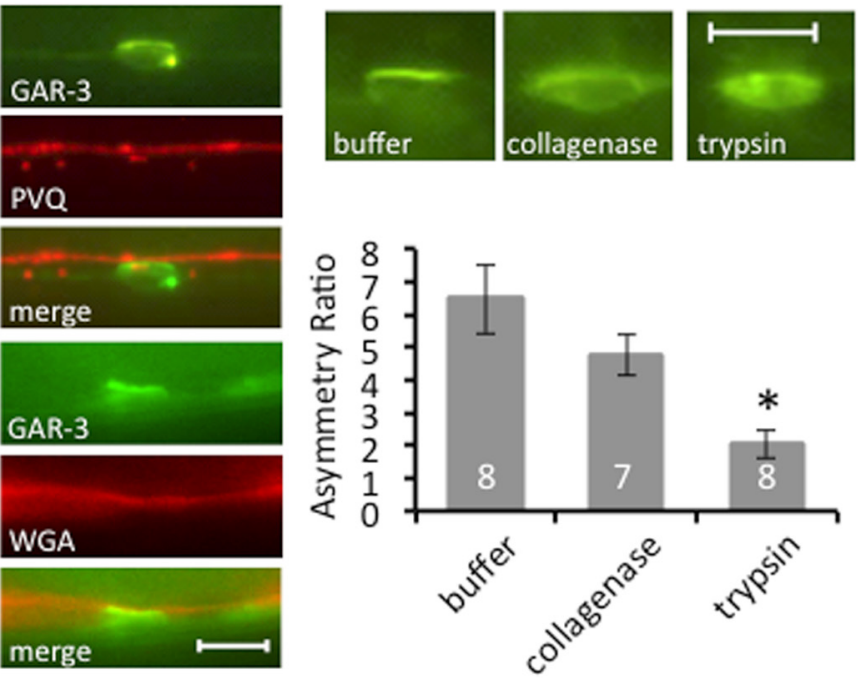

Figure 5. GAR-3 localizes near the extracellular matrix of the basal lamina. $A$, Diagram (top left) of the DA7 motor neuron cell body and cells that it contacts (constructed from White et al., 1976). Red is the hypodermis (skin), green are neuronal processes, and red line is basal lamina. $M$, Medial; $L$, lateral; $D$, dorsal; $V$, ventral. Representative images of GAR-3-GFP in DA/DB motor neurons (vjls50) colocalized with mCherry expressed in DVB (vjEx746[P unc-47::mCherry]), PVQ (vjEx747[P sra-6::mCherry]), AVF (vjEx603[P dfk-2::mCherry]), or AVB (vjEx474[P glr-1::mCherry]) and WGA injected into the pseudocoelomic cavity. $B$, Representative images and quantification of asymmetry of GAR-3-GFP in DA/DB motor neurons in dissected animals following treatment of control (M9), collagenase $(1 \mathrm{~mm})$, and trypsin $(0.05 \%)$ for $1 \mathrm{~min} .{ }^{*} p<0.05$, Student's $t$ tests. Numbers in bars denote the number of animals examined. Scale bar, $5 \mu \mathrm{m}$.

\section{Humoral ACh can activate GAR-3}

The localization of GAR-3 to the basal lamina, which lacks cholinergic synapses, raises the possibility that GAR-3 may be activated by nonsynaptic ACh. To explore this idea, we determined whether ACh released from a source distant from motor neuron cell bodies could activate GAR-3. To do this, we drove ACh release from a subset of cholinergic neurons that do not directly contact the ventral nerve cord using a truncated unc-17 promoter fragment $[$ Punc-17(A)]. Punc-17( $(\Delta)$ expresses specifically in head neurons (Rand, 1989; Tsalik and Hobert, 2003). We first confirmed that Punc-17( $\Delta$ ) expression does not overlap with gar-3 expression in the ventral nerve cord by examining fluorescence patterns of animals coexpressing Punc-17( $\Delta)$-mCherry reporters and GAR-3-gfp. We found that mCherry fluorescence was abundant in neurons of the head and in processes in the lateral cords, but was not observed in the ventral nerve cord (Fig. $7 B$ ).

To drive release of endogenously produced $\mathrm{ACh}$ in these neurons, we overexpressed unc-17, the C. elegans vesicular acetylcholine transporter (VAChT). VAChTs are synaptic vesicleassociated transmembrane proteins and overexpression of VAChT in hippocampal neurons increases ACh release by increasing the quantal content of vesicles (Song et al., 1997; Nagy and Aubert, 2012). We found that expressing unc-17/VAChT under the Punc-17( $\Delta)$ promoter caused aldicarb hypersensitivity (Fig. 7C), implying that the release of ACh from head/lateral neurons by unc-17/VAChT can increase synaptic transmission at NMJs. Similarly, unc-17/VAChT expression under the Punc$17(\Delta)$ promoter significantly increased SPHK-1-GFP abundance at synapses (Fig. $7 D$ ). Both the increases in aldicarb sensitivity and SPHK-1 abundance caused by unc-17/VAChT expression were completely blocked by gar-3 mutations (Fig. $7 C, D$ ). Together, these results indicate that activation of GAR-3 by endogenous ACh released from distant neurons can positively regulate transmitter release form presynaptic motor terminals.

\section{Discussion}

In this study, we show that GAR-3, a C. elegans homolog of the M1/M3/M5 muscarinic receptor family, is preferentially localized on motor neuron cell body membranes that contact the basal lamina of the pseudocoelom. This localization is dependent on the GAR-3 $\mathrm{N}$-terminal extracellular domain and is critical for gar-3-mediated regulation of presynaptic function. These results indicate that specialized membrane localization of $\mathrm{mAChRs}$ in neurons may be an evolutionarily conserved feature of neuromodulation by muscarinic signaling.

\section{The function of asymmetric GAR-3 in motor neuron cell bodies}

Examination of the subcellular localization of GAR-3-GFP in motor neurons showed several striking features. First, GAR-3 was preferentially concentrated on cell bodies and not detectable on dendrites or axons using multicopy overexpression and single-copy insertion of GAR-3-GFP. Second, GAR-3 is likely to be on the surface of the cell, because GAR-3-GFP-expressing animals responded robustly to exogenously applied arecoline, and digestion of the ECM caused rapid redistribution of GAR-3-GFP. Third, GAR-3-GFP adopted an asymmetric localization pattern along the dorsal region of neuronal cell bodies, and mislocalized GAR-3 variants failed to restore proper aldicarb responsiveness and presynaptic SPHK-1 recruitment to gar-3 mutants. Finally, in unc-80 mutants, which have disrupted calcium signal propagation from cell bodies to axons (Yeh et al., 2008), GAR-3 activation failed to regulate SPHK-1 presynaptic abundance.

One model to explain these results is illustrated in Figure $7 E$. ACh in the pseudocoelom acts on GAR-3 on motor neuron cell bodies, which can propagate a signal to axon terminals. This propagation is likely to be dependent on calcium currents mediated by UNC-80/NCA-1/NCA-2. Subsequently, activation of presynaptic voltage gated calcium channels promotes neurotransmitter release in part by regulating the levels of SPHK- 1 at release sites. SPHK-1 is an enzyme that converts sphingosine to 


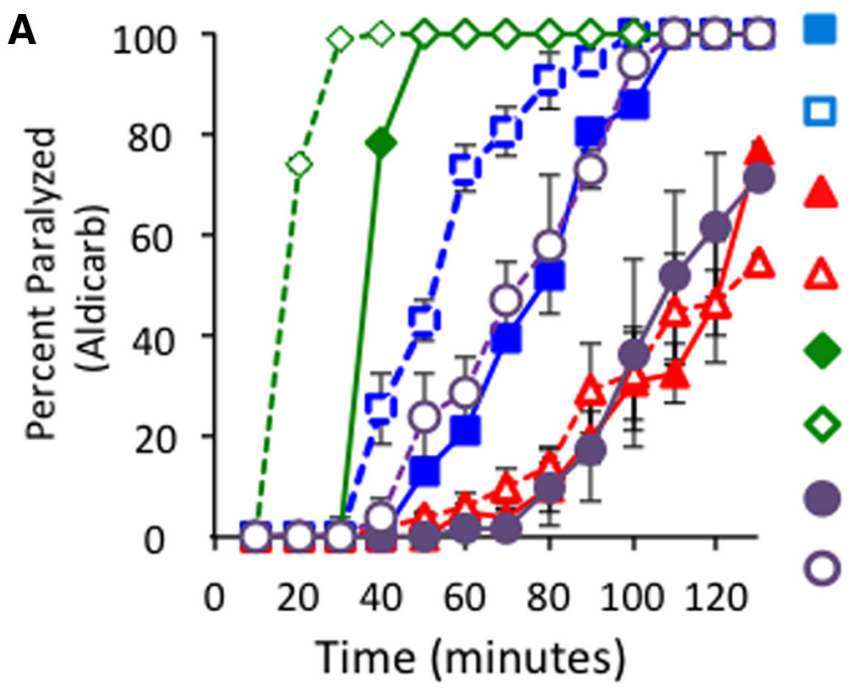

wild type

wild type + arecoline

gar-3

gar- $3+$ arecoline

gar-3; cholinergic GAR-3

gar-3; cholinergic GAR-3 + arecoline

gar-3; cholinergic GAR-1-3

gar-3; cholinergic GAR-1-3 + arecoline

B
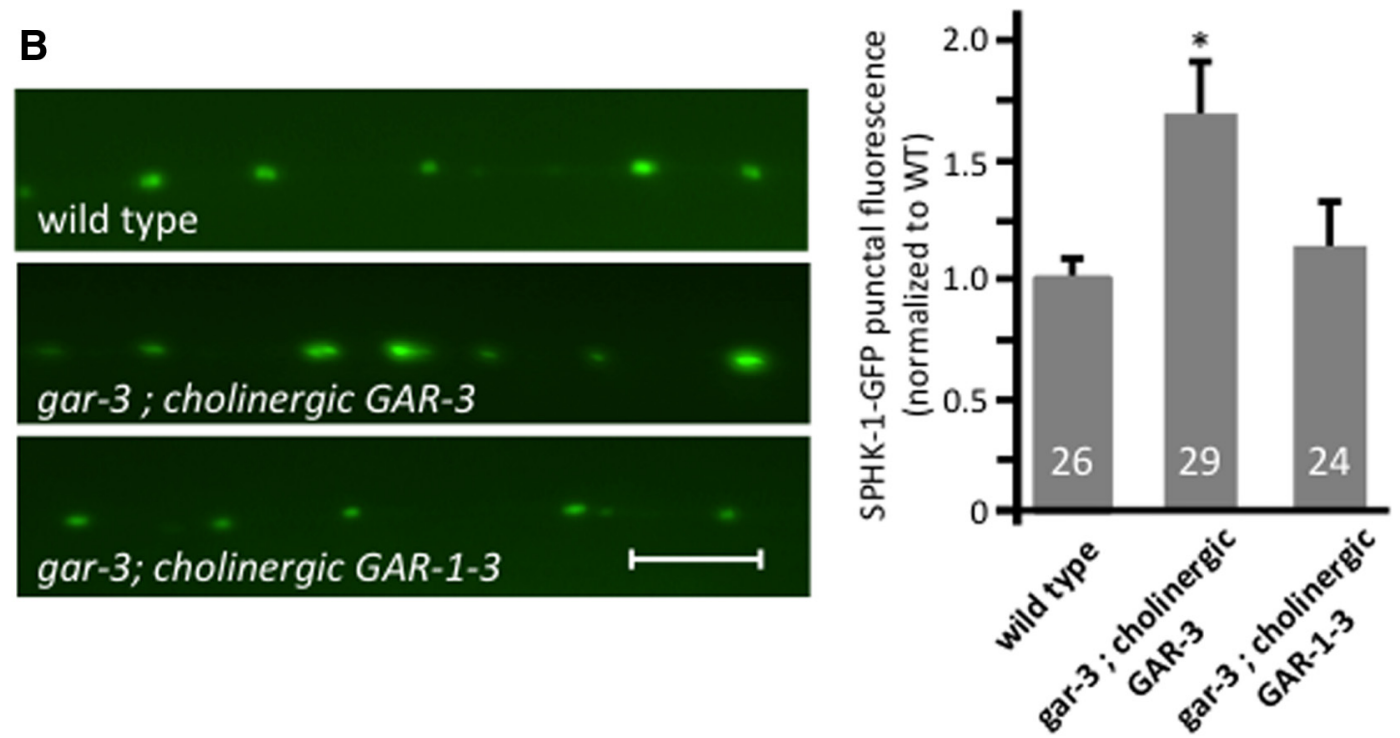

Figure 6. Behavioral and synaptic defects caused by mislocalized GAR-3/mAChR variants. $A$, Rates of worm paralysis of wild-type, gar-3, and gar-3 animals expressing the cholinergic GAR-3 (vjEx635[P unc-17::gar-3b]) or cholinergic GAR-1-3 (vjEx744[P unc-17::GAR-1-3b]) transgenes upon exposure to the acetylcholine esterase inhibitor aldicarb (1.0 mM) following a $2 \mathrm{~h}$ pretreatment with control M9 (solid lines) or arecoline (15 mm in M9; dotted lines). $\boldsymbol{B}$, Representative images and quantification of SPHK-1-GFP punctal fluorescence at synapses of DA/DB motor neurons of gar-3 mutants expressing the indicated transgenes. Numbers in bars denote the number of animals examined, error bars are \pm SEM. Scale bars, $10 \mu \mathrm{m}$. ${ }^{*} p<0.05$, Student's $t$ tests.

sphingosine-1-phosphate, and local generation of sphingosine1 -phosphate at presynaptic terminals is proposed to promote neurotransmitter release (Brailoiu et al., 2002; Chan and Sieburth, 2012; Chan et al., 2012) Genetic and biochemical studies have identified several signaling components that are likely to act downstream of GAR-3 to regulate synaptic SPHK-1 including the heterotrimeric G-protein EGL-30/G $\alpha$ q, the EGL-30 effector UNC-73/Trio, the calcium/calmodulin binding protein CALM$1 / \mathrm{CIB}$, and the voltage gated calcium channel UNC-2 (Chan and Sieburth, 2012; Chan et al., 2012). It remains to be determined where within the motor neurons these components act to transduce the signals from GAR-3 to the synapse. It is also possible that low levels of GAR-3 are found at synapses, below fluorescence detection, and contribute to changes in neurotransmission. Our results also do not rule out the possibility that NCA-1/NCA- 2 may act in parallel to GAR-3 signaling to regulate presynaptic release. Interestingly, the NALCN channel is activated by the M3 mAChR in pancreatic $\beta$ cells, and activation of NALCN appears to be independent of heterotrimeric G-proteins but dependent on the nonreceptor tyrosine kinase Src in $\beta$ cells and in pyramidal cells of the hippocampus (Lu et al., 2009; Swayne et al., 2009).

\section{Mechanisms of asymmetry}

We found that the extracellular N-terminus of GAR-3 was necessary and sufficient for the fivefold enrichment of GAR3-GFP in dorsal neuronal cell bodies and was necessary for membrane localization in muscles. We identified a putative $\mathrm{N}$-linked glycosylation site (N33) within the N-terminal that was partially required for GAR-3-GFP localization. Although the N-terminus of GAR-3 does not share significant overall homology with other C. elegans or mammalian mAChR family members, human M3 and M4 mAChRs have been shown to be glycosylated in vivo (Nathanson, 2008; Romero-Fernandez et al., 2011). In COS cells, M3 mAChR is glycosylated on four Asn residues in its $\mathrm{N}$-terminus, and mutation of these residues 

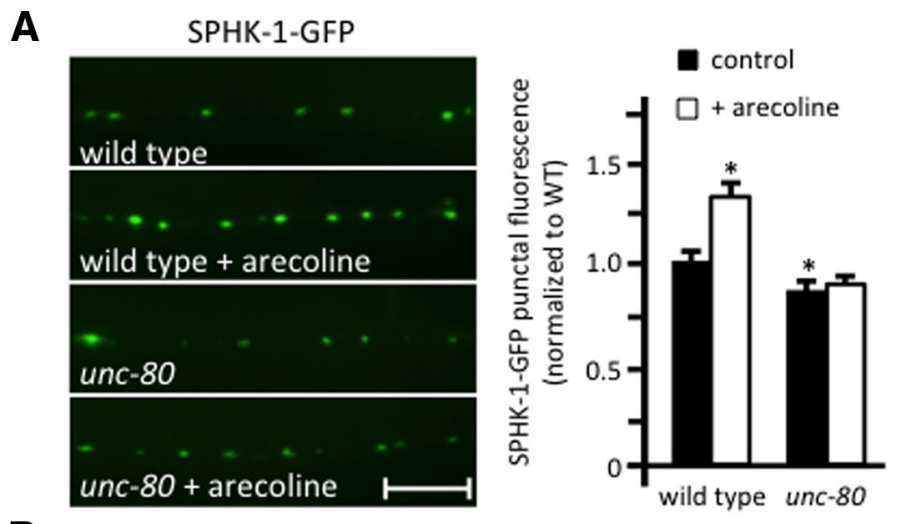

$B$ head tail
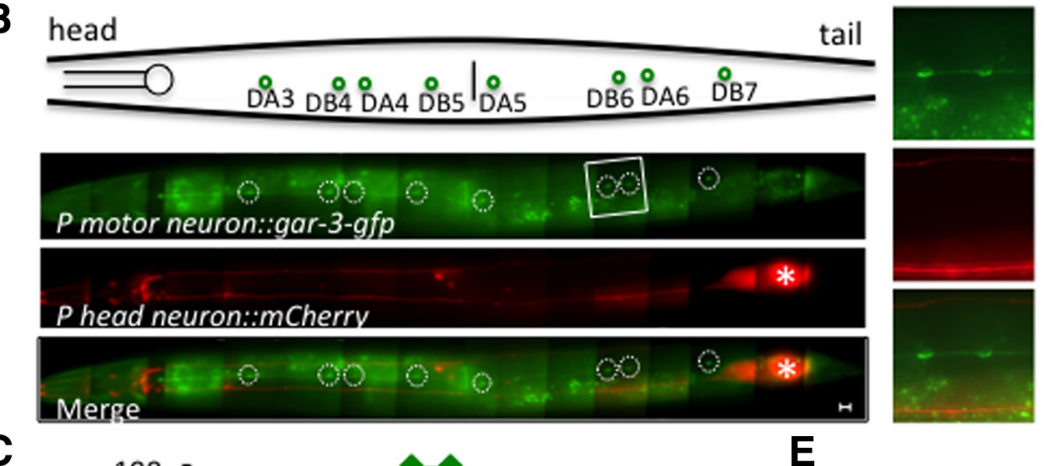

C

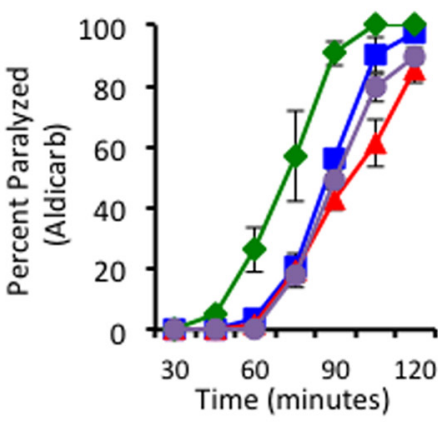

D

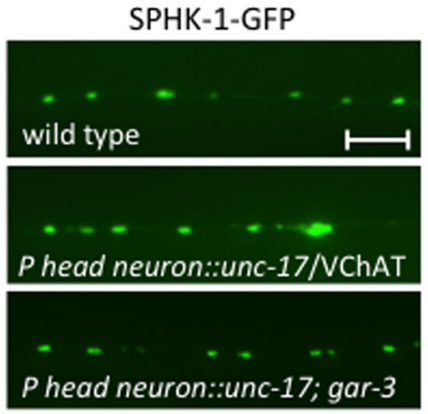

\section{wild type}

A gar-3

P head neuron::

unc-17/VAChT

P head neuron:: unc-17; gar-3

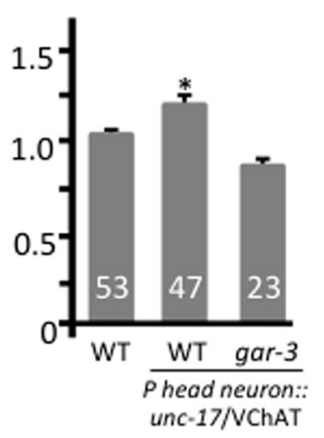

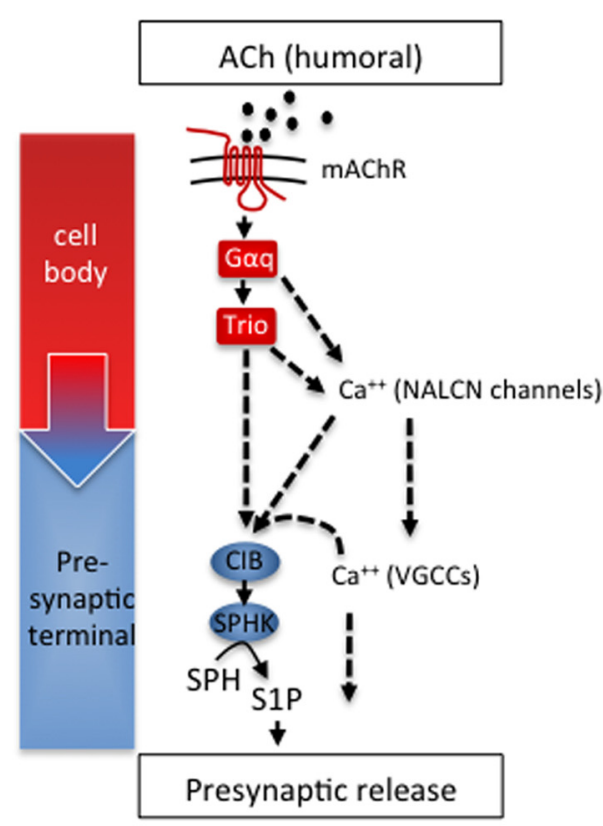

Figure 7. Endogenous ACh released form head neurons causes aldicarb hypersensitivity and recruits SPHK-1 to synapses. $A$, Representative images and quantification of SPHK-1-GFP punctal fluorescence in DA/DB motor neuron axons in the indicated strains treated with either control (M9) or arecoline (15 mm in M9) $2 \mathrm{~h}$ before analysis. $\boldsymbol{B}$, Top, Schematic of DA/DB neuron positions as viewed from the ventral side of the animal. Bottom, Images of animals expressing P motorneuron::GAR-3-gfp (vjls50) or Phead neuron::mCherry (vjEx754). Right, Close-up images of the boxed area. Asterisks denote nonspecific gut fluorescence. $C$, Aldicarb responsiveness of the indicated strains. Head neuron unc-17/VAChT is the vjEx755 transgene. D, Representative images and quantification of SPHK-1-GFP puncta in the indicated genotypes. $E$, Model of the GAR-3 signaling pathway in motor neurons, in which GAR-3 is proposed to be activated by humoral ACh to modulate presynaptic release. Dotted lines denote indirect or unconfirmed interactions. Red is indicative of cell body localization and blue of synaptic localization within cells in the model. CIB, Calcium and integrin binding protein; SPHK, sphingosine kinase; SPH, sphingosine; S1P, sphingosine-1-phosphate; VGCCs, voltage gated calcium channels. Scale bars, $10 \mu \mathrm{m}$. Error bars indicate \pm SEM. ${ }^{*} p<$ 0.05 , Student's $t$ tests.

disrupts transport of the receptor to the plasma membrane (Romero-Fernandez et al., 2011).

We identified OSTD-1/OST- $\delta$ as a candidate for positively regulating GAR- 3 asymmetry. OST- $\delta$ is a component of the OST complex that catalyzes the initial step in the biosynthesis of
$\mathrm{N}$-linked glycoproteins in the lumen of the rough endoplasmic reticulum (Chavan and Lennarz, 2006). OSTD-1/OST- $\delta$ is expressed ubiquitously in C. elegans, including in motor neurons, and knockdown of this gene leads to locomotion defects (www. wormbase.org; Simmer et al., 2003). More studies will help de- 
termine whether OSTD-1 directly participates in the localization of GAR-3 and whether other subunits of this complex also contribute to GAR-3 localization.

Our finding that the third cytoplasmic loop (the i3 loop) of GAR-3 was not necessary for the asymmetric localization of GAR-3 contrasts with the mechanisms by which M2 and M3 $\mathrm{mAChRs}$ are sorted in polarized epithelial cells. In Madin-Darby canine kidney cells, M2 and M3 mAChRs are delivered to the apical and basolateral membranes, respectively, using short sorting signals located in their i3 loops (Nadler et al., 2001; Iverson et al., 2005). It is thought that the i3 loop interacts with cytosolic factors that direct the polarized trafficking of vesicles as they go through the secretory pathway (Nathanson, 2008; Reiner and Nathanson, 2012). Thus, we speculate that GAR-3 localization is not regulated at the level of vesicle trafficking but rather by an interaction between the $\mathrm{N}$-terminal extracellular domain and the ECM once GAR-3 is delivered to the cell surface.

Based on our colocalization studies, we believe that the $\mathrm{N}$-terminus of GAR-3 interacts with a component of the basal lamina. NID-1/Nidogen and CLE-1/type XVIII collagen are components of the basal lamina surrounding cell bodies of motor neurons, and both proteins are proposed to regulate synaptic development (Kramer, 1994; Ackley et al., 2003). However, these proteins are enriched in lateral regions of the basal lamina that are not in contact with motor neuron cell bodies (Kramer, 1994; Ackley et al., 2003), and mutations in the genes encoding these proteins did not alter GAR-3-GFP localization (D. Sieburth, unpublished observations), making it unlikely that they are involved in GAR-3 localization. A member of the adhesion GPCR family, GPR56, binds collagen III at its N-terminal extracellular domain, and this interaction is critical for GPR56 activation and proper cell surface expression (Jin et al., 2007; Singer et al., 2013). Interestingly GAR-3 residues $38-48$ show $82 \%$ similarity with residues in the GPR56 collagen-binding site. However, the GPR56 collagen-binding site did not confer localization to GAR-3 lacking its N-terminus (D. Sieburth, unpublished observations). Identifying components of the basal lamina that regulate GAR-3 localization will help elucidate mechanisms underlying the establishment and maintenance of GAR-3 in cell bodies.

\section{Nonsynaptic ACh activates extrasynaptic GAR-3}

Cholinergic transmission in the brain can occur by wired transmission, mediated by ACh released into the synaptic cleft, or by volume transmission by ambient ACh (Sarter et al., 2009; Agnati et al., 2010; Trueta and De-Miguel, 2012). Cholinergic signaling through volume transmission is supported by observations that there are fewer cholinergic fibers than cells expressing AChRs. Also, cholinergic neurons make few synaptic specializations with target cells and M3 receptors in cortical and hippocampal neurons are largely extrasynaptic (Descarries and Mechawar, 2000). Our results argue that GAR-3 is activated by extrasynaptic ACh released from distant cells, supporting a role for volume transmission in mediating motor function. First, GAR-3 localized to extrasynaptic regions of motor neuron cell bodies that are devoid of cholinergic synaptic contacts, as shown by electron microscopy (White et al., 1976). Second, colocalization studies showed that GAR-3 is exposed to the basal lamina, an ECM that separates motor neuron cell bodies from the pseudocoelom. Third, driving ACh release from cholinergic neurons in the head and lateral ganglia (using a truncated unc-17 promoter) was sufficient for
GAR-3-dependent effects on NMJ protein composition and function. Because motor neurons receive direct synaptic input from command interneurons, but not cholinergic neurons of the head or lateral cords, the ACh released from these cells is likely to activate GAR-3 via an indirect mechanism.

What is the cellular source of ACh that activates GAR-3 on motor neurons? The pseudocoelom is a body cavity of the worm that supplies gases, nutrients, and other molecules to tissues throughout the animal, and indirect evidence supports the idea that ACh may be present at low levels in the pseudocoelom. First, a putative ACh gated ion channel, cup-4, functions in scavenger cells that are in direct contact with the pseudocoelom (Patton et al., 2005). Second, the choline transporter, cho- 1 , which mediates the re-uptake of the breakdown product of ACh, may be expressed in noncholinergic cell types including the intestine, which is exposed to the pseudocoelom (Mullen et al., 2007; Duerr et al., 2008). Finally, numerous sensory and interneurons are in contact with the pseudocoelom (Cheung et al., 2004; Kao et al., 2007), raising the possibility that certain cholinergic neurons in the head and tail may also release ACh into the pseudocoelom. gar-3 mutants have weak aldicarb resistance phenotypes and no detectable defect in SPHK-1 recruitment (Chan et al., 2012), suggesting that under normal conditions, ACh levels may be low. Interestingly, the binding affinities of agonists to GAR-3 and to M1 mAChRs (Park et al., 2006), which mediate volume transmission in the murine hippocampus (Yamasaki et al., 2010), are comparable. Perhaps the highly concentrated localization of GAR-3 at the basal lamina maximizes response to low ACh levels in the pseudocoelom. Interestingly, humoral transmission by other neuromodulators is proposed to control motor neuron activity in C. elegans: dopamine receptors controlling locomotion are not postsynaptic to any dopaminergic neuron terminal (Chase et al., 2004), and serotonin receptors mediating learned feeding responses to familiar foods are found far from the primary serotonin release sites (Song et al., 2013). Humoral transmission may provide a means to mediate modulatory aspects of neuronal networks on slower timescales (Sarter et al., 2009; Agnati et al., 2010; Fuxe et al., 2010). Indeed, our data support a modulatory function of GAR-3 in the regulation of neurotransmission because the functional defects of gar-3 mutants are more obvious under activity-dependent states (e.g., arecoline treatment, unc-17/VChAT overexpression). Identifying the cellular source of ACh that activates GAR-3 signaling in motor neurons will help to clarify the role that muscarinic signaling plays in regulating behavior.

\section{References}

Ackley BD, Kang SH, Crew JR, Suh C, Jin Y, Kramer JM (2003) The basement membrane components nidogen and type XVIII collagen regulate organization of neuromuscular junctions in Caenorhabditis elegans. J Neurosci 23:3577-3587. Medline

Agnati LF, Guidolin D, Guescini M, Genedani S, Fuxe K (2010) Understanding wiring and volume transmission. Brain Res Rev 64:137-159. CrossRef Medline

Brailoiu E, Cooper RL, Dun NJ (2002) Sphingosine 1-phosphate enhances spontaneous transmitter release at the frog neuromuscular junction. Br J Pharmacol 136:1093-1097. CrossRef Medline

Chan JP, Sieburth D (2012) Localized sphingolipid signaling at presynaptic terminals is regulated by calcium influx and promotes recruitment of priming factors. J Neurosci 32:17909-17920. CrossRef Medline

Chan JP, Hu Z, Sieburth D (2012) Recruitment of sphingosine kinase to presynaptic terminals by a conserved muscarinic signaling pathway promotes neurotransmitter release. Genes Dev 26:1070-1085. CrossRef Medline

Chase DL, Pepper JS, Koelle MR (2004) Mechanism of extrasynaptic dopa- 
mine signaling in Caenorhabditis elegans. Nat Neurosci 7:1096-1103. CrossRef Medline

Chavan M, Lennarz W (2006) The molecular basis of coupling of translocation and $N$-glycosylation. Trends Biochem Sci 31:17-20. CrossRef Medline

Cheung BH, Arellano-Carbajal F, Rybicki I, de Bono M (2004) Soluble guanylate cyclases act in neurons exposed to the body fluid to promote $C$. elegans aggregation behavior. Curr Biol 14:1105-1111. CrossRef Medline

Ch'ng Q, Sieburth D, Kaplan JM (2008) Profiling synaptic proteins identifies regulators of insulin secretion and lifespan. PLoS Genet 4:e1000283. CrossRef Medline

Descarries L, Mechawar N (2000) Ultrastructural evidence for diffuse transmission by monoamine and acetylcholine neurons of the central nervous system. Prog Brain Res 125:27-47. CrossRef Medline

Dittman JS, Kaplan JM (2006) Factors regulating the abundance and localization of synaptobrevin in the plasma membrane. Proc Natl Acad Sci U S A 103:11399-11404. CrossRef Medline

Dittman JS, Kaplan JM (2008) Behavioral impact of neurotransmitteractivated G-protein-coupled receptors: muscarinic and GABAB receptors regulate Caenorhabditis elegans locomotion. J Neurosci 28:7104-7112. CrossRef Medline

Duerr JS, Han HP, Fields SD, Rand JB (2008) Identification of major classes of cholinergic neurons in the nematode Caenorhabditis elegans. J Comp Neurol 506:398-408. CrossRef Medline

Eglen RM (2006) Muscarinic receptor subtypes in neuronal and nonneuronal cholinergic function. Auton Autacoid Pharmacol 26:219-233. CrossRef Medline

Fuxe K, Dahlström AB, Jonsson G, Marcellino D, Guescini M, Dam M, Manger P, Agnati L (2010) The discovery of central monoamine neurons gave volume transmission to the wired brain. Prog Neurobiol 90:82-100. CrossRef Medline

Fuxe K, Borroto-Escuela DO, Romero-Fernandez W, Diaz-Cabiale Z, Rivera A, Ferraro L, Tanganelli S, Tarakanov AO, Garriga P, Narváez JA, Ciruela F, Guescini M, Agnati LF (2012) Extrasynaptic neurotransmission in the modulation of brain function. Focus on the striatal neuronal-glial networks. Front Physiol 3:136. CrossRef Medline

Green JL, Inoue T, Sternberg PW (2008) Opposing Wnt pathways orient cell polarity during organogenesis. Cell 134:646-656. CrossRef Medline

Hardin J, King RS (2008) The long and the short of Wnt signaling in C. elegans. Curr Opin Genet Dev 18:362-367. CrossRef Medline

Iverson HA, Fox D 3rd, Nadler LS, Klevit RE, Nathanson NM (2005) Identification and structural determination of the $\mathrm{M}(3)$ muscarinic acetylcholine receptor basolateral sorting signal. J Biol Chem 280: 24568-24575. CrossRef Medline

Jin Z, Tietjen I, Bu L, Liu-Yesucevitz L, Gaur SK, Walsh CA, Piao X (2007) Disease-associated mutations affect GPR56 protein trafficking and cell surface expression. Hum Mol Genet 16:1972-1985. CrossRef Medline

Kamath RS, Martinez-Campos M, Zipperlen P, Fraser AG, Ahringer J (2001) Effectiveness of specific RNA-mediated interference through ingested double-stranded RNA in Caenorhabditis elegans. Genome Biol 2:research0002. CrossRef Medline

Kao G, Nordenson C, Still M, Rönnlund A, Tuck S, Naredi P (2007) ASNA-1 positively regulates insulin secretion in C. elegans and mammalian cells. Cell 128:577-587. CrossRef Medline

Kramer JM (1994) Genetic analysis of extracellular matrix in C. elegans. Annu Rev Genet 28:95-116. CrossRef Medline

Lackner MR, Nurrish SJ, Kaplan JM (1999) Facilitation of synaptic transmission by EGL-30 Gqalpha and EGL-8 PLCbeta: DAG binding to UNC-13 is required to stimulate acetylcholine release. Neuron 24:335-346. CrossRef Medline

Leach K, Simms J, Sexton PM, Christopoulos A (2012) Structure-function studies of muscarinic acetylcholine receptors. Handb Exp Pharmacol 208: 29-48. CrossRef Medline

Levey AI, Kitt CA, Simonds WF, Price DL, Brann MR (1991) Identification and localization of muscarinic acetylcholine receptor proteins in brain with subtype-specific antibodies. J Neurosci 11:3218-3226. Medline

Lewis JA, Wu CH, Berg H, Levine JH (1980) The genetics of levamisole resistance in the nematode Caenorhabditis elegans. Genetics 95:905-928. Medline

Liles WC, Nathanson NM (1986) Regulation of neuronal muscarinic acetylcholine receptor number by protein glycosylation. J Neurochem 46:89-95. CrossRef Medline
Liu Y, LeBoeuf B, Garcia LR (2007) G alpha(q)-coupled muscarinic acetylcholine receptors enhance nicotinic acetylcholine receptor signaling in Caenorhabditis elegans mating behavior. J Neurosci 27:1411-1421. CrossRef Medline

Lu B, Su Y, Das S, Wang H, Wang Y, Liu J, Ren D (2009) Peptide neurotransmitters activate a cation channel complex of NALCN and UNC80. Nature 457:741-744. CrossRef Medline

Mahoney TR, Luo S, Nonet ML (2006) Analysis of synaptic transmission in Caenorhabditis elegans using an aldicarb-sensitivity assay. Nat Protoc 1:1772-1777. CrossRef Medline

Mello CC, Kramer JM, Stinchcomb D, Ambros V (1991) Efficient gene transfer in C. elegans: extrachromosomal maintenance and integration of transforming sequences. EMBO J 10:3959-3970. Medline

Miller KG, Alfonso A, Nguyen M, Crowell JA, Johnson CD, Rand JB (1996) A genetic selection for Caenorhabditis elegans synaptic transmission mutants. Proc Natl Acad Sci U S A 93:12593-12598. CrossRef Medline

Mrzljak L, Levey AI, Goldman-Rakic PS (1993) Association of m1 and $\mathrm{m} 2$ muscarinic receptor proteins with asymmetric synapses in the primate cerebral cortex: morphological evidence for cholinergic modulation of excitatory neurotransmission. Proc Natl Acad Sci U S A 90:5194-5198. CrossRef Medline

Mullen GP, Mathews EA, Vu MH, Hunter JW, Frisby DL, Duke A, Grundahl K, Osborne JD, Crowell JA, Rand JB (2007) Choline transport and de novo choline synthesis support acetylcholine biosynthesis in Caenorhabditis elegans cholinergic neurons. Genetics 177:195-204. CrossRef Medline

Nadler LS, Kumar G, Nathanson NM (2001) Identification of a basolateral sorting signal for the M3 muscarinic acetylcholine receptor in MadinDarby canine kidney cells. J Biol Chem 276:10539-10547. CrossRef Medline

Nagy PM, Aubert I (2012) Overexpression of the vesicular acetylcholine transporter increased acetylcholine release in the hippocampus. Neuroscience 218:1-11. CrossRef Medline

Nance J, Zallen JA (2011) Elaborating polarity: PAR proteins and the cytoskeleton. Development 138:799-809. CrossRef Medline

Nathanson NM (2008) Synthesis, trafficking, and localization of muscarinic acetylcholine receptors. Pharmacol Ther 119:33-43. CrossRef Medline

Oldenburg IA, Ding JB (2011) Cholinergic modulation of synaptic integration and dendritic excitability in the striatum. Curr Opin Neurobiol 21: 425-432. CrossRef Medline

Park YS, Cho TJ, Cho NJ (2006) Stimulation of cyclic AMP production by the Caenorhabditis elegans muscarinic acetylcholine receptor GAR-3 in Chinese hamster ovary cells. Arch Biochem Biophys 450:203-207. CrossRef Medline

Patton A, Knuth S, Schaheen B, Dang H, Greenwald I, Fares H (2005) Endocytosis function of a ligand-gated ion channel homolog in Caenorhabditis elegans. Curr Biol 15:1045-1050. CrossRef Medline

Picciotto MR, Higley MJ, Mineur YS (2012) Acetylcholine as a neuromodulator: cholinergic signaling shapes nervous system function and behavior. Neuron 76:116-129. CrossRef Medline

Rand JB (1989) Genetic analysis of the cha-1-unc-17 gene complex in Caenorhabditis. Genetics 122:73-80. Medline

Reiner C, Nathanson NM (2012) Muscarinic receptor trafficking. Handb Exp Pharmacol 208:61-78. CrossRef Medline

Richmond JE (2006) Electrophysiological recordings from the neuromuscular junction of C. elegans. WormBook 1-8. CrossRef Medline

Ritter SL, Hall RA (2009) Fine-tuning of GPCR activity by receptorinteracting proteins. Nat Rev Mol Cell Biol 10:819-830. CrossRef Medline

Romero-Fernandez W, Borroto-Escuela DO, Alea MP, Garcia-Mesa Y, Garriga $P$ (2011) Altered trafficking and unfolded protein response induction as a result of M3 muscarinic receptor impaired $\mathrm{N}$-glycosylation. Glycobiology 21:1663-1672. CrossRef Medline

Rouse ST, Thomas TM, Levey AI (1997) Muscarinic acetylcholine receptor subtype, $\mathrm{m} 2$ : diverse functional implications of differential synaptic localization. Life Sci 60:1031-1038. CrossRef Medline

Rouse ST, Gilmor ML, Levey AI (1998) Differential presynaptic and postsynaptic expression of $\mathrm{m} 1-\mathrm{m} 4$ muscarinic acetylcholine receptors at the perforant pathway/granule cell synapse. Neuroscience 86:221-232. CrossRef Medline

Rouse ST, Edmunds SM, Yi H, Gilmor ML, Levey AI (2000) Localization of $\mathrm{M}(2)$ muscarinic acetylcholine receptor protein in cholinergic and 
noncholinergic terminals in rat hippocampus. Neurosci Lett 284:182186. CrossRef Medline

Sarter M, Parikh V, Howe WM (2009) Phasic acetylcholine release and the volume transmission hypothesis: time to move on. Nat Rev Neurosci 10:383-390. CrossRef Medline

Simmer F, Moorman C, van der Linden AM, Kuijk E, van den Berghe PV, Kamath RS, Fraser AG, Ahringer J, Plasterk RH (2003) Genome-wide RNAi of C. elegans using the hypersensitive rrf-3 strain reveals novel gene functions. PLoS Biol 1:E12. CrossRef Medline

Singer K, Luo R, Jeong SJ, Piao X (2013) GPR56 and the developing cerebral cortex: cells, matrix, and neuronal migration. Mol Neurobiol 47:186-196. CrossRef Medline

Song BM, Faumont S, Lockery S, Avery L (2013) Recognition of familiar food activates feeding via an endocrine serotonin signal in Caenorhabditis elegans. Elife 2:e00329. CrossRef Medline

Song H, Ming G, Fon E, Bellocchio E, Edwards RH, Poo M (1997) Expression of a putative vesicular acetylcholine transporter facilitates quantal transmitter packaging. Neuron 18:815-826. CrossRef Medline

Steger KA, Avery L (2004) The GAR-3 muscarinic receptor cooperates with calcium signals to regulate muscle contraction in the Caenorhabditis elegans pharynx. Genetics 167:633-643. CrossRef Medline

Swayne LA, Mezghrani A, Varrault A, Chemin J, Bertrand G, Dalle S, Bourinet E, Lory P, Miller RJ, Nargeot J, Monteil A (2009) The NALCN ion channel is activated by M3 muscarinic receptors in a pancreatic beta-cell line. EMBO Rep 10:873-880. CrossRef Medline

Trueta C, De-Miguel FF (2012) Extrasynaptic exocytosis and its mecha- nisms: a source of molecules mediating volume transmission in the nervous system. Front Physiol 3:319. CrossRef Medline

Tsalik EL, Hobert O (2003) Functional mapping of neurons that control locomotory behavior in Caenorhabditis elegans. J Neurobiol 56:178-197. CrossRef Medline

Vizi ES, Fekete A, Karoly R, Mike A (2010) Non-synaptic receptors and transporters involved in brain functions and targets of drug treatment. Br J Pharmacol 160:785-809. CrossRef Medline

Wess J, Eglen RM, Gautam D (2007) Muscarinic acetylcholine receptors: mutant mice provide new insights for drug development. Nat Rev Drug Discov 6:721-733. CrossRef Medline

White JG, Southgate E, Thomson JN, Brenner S (1976) The structure of the ventral nerve cord of Caenorhabditis elegans. Philos Trans R Soc Lond B Biol Sci 275:327-348. Medline

Yamasaki M, Matsui M, Watanabe M (2010) Preferential localization of muscarinic M1 receptor on dendritic shaft and spine of cortical pyramidal cells and its anatomical evidence for volume transmission. J Neurosci 30:4408-4418. CrossRef Medline

Yeh E, Ng S, Zhang M, Bouhours M, Wang Y, Wang M, Hung W, Aoyagi K, Melnik-Martinez K, Li M, Liu F, Schafer WR, Zhen M (2008) A putative cation channel, NCA-1, and a novel protein, UNC-80, transmit neuronal activity in C. elegans. PLoS Biol 6:e55. CrossRef Medline 